This item was submitted to Loughborough's Research Repository by the author.

Items in Figshare are protected by copyright, with all rights reserved, unless otherwise indicated.

\title{
Optimisation of the structural modes of automotive-type panels using line stiffeners and point masses to achieve weak acoustic radiation
}

PLEASE CITE THE PUBLISHED VERSION

http://dx.doi.org/10.1016/j.apacoust.2015.01.001

\section{PUBLISHER}

(c) Elsevier Ltd.

\section{VERSION}

AM (Accepted Manuscript)

\section{PUBLISHER STATEMENT}

This work is made available according to the conditions of the Creative Commons Attribution-NonCommercialNoDerivatives 4.0 International (CC BY-NC-ND 4.0) licence. Full details of this licence are available at: https://creativecommons.org/licenses/by-nc-nd/4.0/

\section{LICENCE}

CC BY-NC-ND 4.0

\section{REPOSITORY RECORD}

Rousounelos, Andreas, Stephen J. Walsh, Victor V. Krylov, and Jane L. Horner. 2015. "Optimisation of the Structural Modes of Automotive-type Panels Using Line Stiffeners and Point Masses to Achieve Weak Acoustic Radiation". figshare. https://hdl.handle.net/2134/17273. 


\title{
Optimisation of the structural modes of automotive-type panels using line stiffeners and point masses to achieve weak acoustic radiation
}

\author{
A. Rousounelos, S.J. Walsh, V.V. Krylov and J.L. Horner \\ Department of Aeronautical \& Automotive Engineering, \\ Loughborough University, Loughborough LE11 3TU, UK \\ Corresponding author. \\ Dr. S.J. Walsh \\ E-mail address: S.J.Walsh@Lboro.ac.uk (S.J. Walsh)
}

Keywords: structural-acoustic optimisation; plates; automotive-type panels; stiffeners; genetic algorithm. 


\section{ABSTRACT}

In this paper, an optimisation method is presented that turns the structural modes of an automotive-type panel into weak acoustic radiators. The advantage of the proposed method is that the optimum design does not depend upon a specific excitation mechanism. Hence, the optimised panel can be used in the early stages of vehicle product design when specific knowledge about the excitation forces is not available. In the proposed method the boundary conditions of the panel are specified such that the panel can be considerd in isolation from the rest of the structure. The optimisation of the structural modes of the panel is then achieved by placing a number of constraint masses and stiffeners of optimum size and weight at optimum locations on the plate. Firstly, a theoretical model of a simply-supported plate is developed that takes into account the constraints at arbitrary positions and orientations. Then a genetic algorithm is used to minimise the sound power radiated by different modes on the plate by finding the optimum design for the constraints. Secondly, the use of added masses and stiffeners is then applied to a floor panel of a simplified model of a vehicle body structure in order to reduce the radiated sound. Predicted and experimental results of the radiated sound power from a reference flat panel and from various optimised panel designs are presented to illustrate the effectiveness of the optimised designs in reducing radiated sound from the structure. A combination of masses and stiffeners is shown to be an effective way of constructing weakly radiating structural modes on the panel. 


\section{Introduction}

Structural-acoustic design to achieve low levels of noise radiation from vehicles and machines is a highly complex problem. For this reason, one approach to the design problem is to use automated optimisation algorithms. This approach has been used successfully for the last two decades. In Ref. [1] Marburg presented a review of a large number of publications on structural-acoustic optimisation covering a variety of applications. The merits of different objective functions and design variables were also discussed. Another review paper by Christensen et al [2] discussed the common structural acoustic analysis methods, as well as, design sensitivity analysis methods. Lamancusa [3] presented a study on the numerical optimisation of a rectangular plate excited by a point force. The design variable was the thickness distribution of the plate. Many different objective functions were presented and their merits discussed. From the results shown in Ref. [3] it was clear that the choice of an appropriate objective function was very important for the success of the optimisation procedure. It was also shown that the optimisation results depended strongly on the location of the excitation. Thus, an accurate description of the excitation mechanism would be required for practical applications.

Structural-acoustic problems can be broadly divided into internal and external noise radiation problems. For an internal noise problem the most widely used objective function is a function of the sound pressure at a point of interest in the enclosed space. For example, for a vehicle interior noise optimisation, this point is typically the location of the driver's or passenger's ears. In Ref. [4], Marburg used a sound pressure related exponential objective function to reduce the noise transfer function inside a vehicle cabin by optimising the design of the dashboard. For external noise problems the most widely used acoustic objective function is the sound power radiated by the structure.

Since structural-acoustic problems require high computational power, gradient based optimisers have been extensively introduced over the last decade. However, Hambric [5] suggests that local gradient-based minimisers are not appropriate. In Ref. [5], Hambric presented approximate expressions for a noise radiation function that was used with a simulated annealing algorithm to minimise the noise radiating from a shell structure by the placement of parallel ribs. 
A different optimisation approach was described in Ref. [6] by Koopmann and Fahnline. Their approach attempted to modify the design of the structure in order to ensure that the structural modes only radiated sound weakly. The proposed method had the advantage that the optimisation did not depend on the way the structure was excited. Thus, once the weakly radiating modes have been imposed onto the structure they should be insensitive to the majority of input forces. Hence, once a plate of given geometrical and physical properties has been designed to have weakly radiating modes in a certain frequency range, this plate can be used without having to take into account the possible pressence of complex excitation mechanisms. This method is especially useful in the early stages of the vehicle product design life cycle.

A number of studies using this optimisation method have been published. Wodtke and Koopmann [7] used the Rayleigh-Ritz method for the structural analysis of a clamped rectangular plate with added masses to optimise one specific mode of the structure. For the acoustic analysis the volume velocity of the structure was used as a low-frequency sound power approximation. The design variable was the weight of the added masses whereas their location was considered fixed. The results showed a significant reduction in the radiated sound for the optimised mode. Pierre and Koopmann [8] presented results for optimisation of the first and third modes of a clamped plate using four and nine masses at fixed locations. The finite element method (FEM) was used for the structural analysis and a full calculation was made of the sound power. More recently, this approach has been applied to minimise the sound radiation from rectangular panels with dome-shaped indentations [9].

A classic publication that discusses the effect of local modification upon the response vibration of a linear system is that by Weissenburger [10]. In Ref. [10] the response of a modified system was given in terms of the eigenfunctions of the unmodified system, thus, simplifying the analysis. Wu and Luo [11] used this principle for the vibration analysis of a rectangular plate with attached point masses and springs.

The novelty of the present paper is that the theoretical analysis presented in Refs. $[10,11]$ is extended so as to include the effect of added stiffeners, instead of springs, at arbitrary locations and orientations on the plate. This is then combined with the optimisation approach presented in Refs. [6-8]. Thus, the previous knowledge is extended in two ways. Firstly, stiffeners, in addition to masses, are used to design the weakly radiating plate modes. Secondly, the geometric properties as 
well as the locations of the added masses and stiffeners are optimised. To demonstrate this new approach, predicted and experimentally measured results are presented for a simply-supported plate with different configuations of added masses and stiffeners. As an automotive case study, the proposed approach is then applied numericaly to an 'isolated' panel of a simplified vehicle model.

\section{Theoretical model of a simply-supported plate with added point masses and line stiffeners}

\subsection{Structural model}

The effect of constraints, such as attached masses and stiffeners, can be introduced into the equation of motion for flexural vibrations of an unconstrained plate of uniform thickness by adding forcing terms in the right-hand side of the equation which then represent the constraint forces:

$$
\begin{aligned}
D \nabla^{4} w(x, y, t)+\rho_{p} h_{p} \ddot{w}(x, y, t) & =-\sum_{l} m_{l} \ddot{w}(x, y, t) M_{l}(x, y) \\
& \quad-\sum_{s}\left[E I_{s} \nabla^{4} w(x, y, t)+\rho_{s, b} h_{s, b} \ddot{w}(x, y, t)\right] S_{s}(x, y),
\end{aligned}
$$

where $w$ is the transverse deflection of the plate, $\rho_{p}$ is the material density, $D$ the flexural rigidity and $h_{p}$ is the thickness of the plate. For point mass and beamstiffener constraints $m_{l}$ is the weight of the $l^{\text {th }}$ mass and $E I_{s}$ and $\rho_{s, b} h_{s, b}$ are the bending stiffness and surface density of the $s^{\text {th }}$ beam-stiffener, respectively. $M_{l}(x, y)$ and $S_{s}(x, y)$ are functions which represent the location of the masses and stiffeners, respectively. For point masses and line stiffeners these function are given by

$$
M_{l}(x, y)=\delta\left(x-x_{l}\right) \delta\left(y-y_{l}\right) \text { and } S_{s}(x, y)= \begin{cases}\delta\left(x-f_{s}(y)\right) & y 1_{s}<y<y 2_{s} \\ 0 & \text { otherwise }\end{cases}
$$


where $\delta(x)$ is the Dirac delta function, $x_{l}, y_{l}$ are the coordinates of the $l^{\text {th }}$ mass and $f_{s}(y)$ is the function of the line of the $s^{\text {th }}$ stiffener starting at coordinates $\left(x 1_{s}, y 1_{s}\right)$ and finishing at coordinates $\left(x 2_{s}, y 2_{s}\right)$. The line function of the $s^{\text {th }}$ stiffener, $f_{s}(y)$, is given by

$$
f_{s}(y)=\frac{y-\beta_{s}}{\alpha_{s}}
$$

where $\alpha_{s}$ and $\beta_{s}$ are determined by solving the linear equation using the starting point and end point coordinates

$$
\alpha_{s}=\frac{y 2_{s}-y 1_{s}}{x 2_{s}-x 1_{s}}, \quad \beta_{s}=y 1_{s}-\frac{\left(y 2_{s}-y 1_{s}\right)}{x 2_{s}-x 1_{s}} x 1_{s} .
$$

Note that the influence of the rotation of the beam-stiffener has been neglected. Using modal superposition for a simply supported rectangular plate the solution of Eq. (1) is given in the form

$$
w(x, y, t)=\sum_{i=1}^{N_{x}} \sum_{j=1}^{N_{y}} W_{i j}(x, y) q_{i j}(t)
$$

with

$$
W_{i j}(x, y)=\sin \frac{i \pi x}{L_{x}} \sin \frac{j \pi y}{L_{y}}
$$

Substituting Eq. (3) into Eq. (1), multiplying each term by $W_{r b}(x, y)$, integrating each term over the surface of the plate and dividing by $\rho_{p} h L_{x} L_{y} / 4$ gives [12]

$$
\omega_{r b}^{2} q_{r b}+\ddot{q}_{r b}+\sum_{i=1}^{N_{x}} \sum_{j=1}^{N_{y}} A_{i j, r b} \ddot{q}_{i j}+\sum_{i=1}^{N_{x}} \sum_{j=1}^{N_{y}} B_{i j, r b} q_{i j}+\sum_{i=1}^{N_{x}} \sum_{j=1}^{N_{y}} C_{i j, r b} \ddot{q}_{i j}=0
$$


where

$\omega_{r b}=\left(\left(\frac{r \pi}{L_{x}}\right)^{2}+\left(\frac{b \pi}{L_{y}}\right)^{2}\right) \sqrt{\frac{D}{\rho_{p} h}}$,

are the natural frequencies in radians per second of the unconstraint plate. $A_{i j, r b}$ is a function of the point masses, $B_{i j, r b}$ is a function of the stiffness of the line stiffeners and $C_{i j, r b}$ is a function of the mass of the line stiffeners. These are defined as

$A_{i j, r b}=\sum_{l} \frac{4 m_{l}}{L_{x} L_{y} \rho_{p} h_{p}} W_{i j}\left(x_{l}, y_{l}\right) W_{r b}\left(x_{l}, y_{l}\right)$,

$B_{i j, r b}=\sum_{s} \frac{4 E I_{s}}{L_{x} L_{y} \rho_{p} h_{p}} \int_{y 1}^{y 2} \frac{d^{4}}{d y^{4}} W_{i j}\left(f_{s}(y), y\right) W_{r b}\left(f_{s}(y), y\right) \mathrm{d} y$,

$C_{i j, r b}=\sum_{s} \frac{4 \rho_{s, b} h_{s, b}}{L_{x} L_{y} \rho_{p} h_{p}} \int_{y 1}^{y 2} W_{i j}\left(f_{s}(y), y\right) W_{r b}\left(f_{s}(y), y\right) \mathrm{d} y$.

To reduce the number of indices in Eq. (4), new indices $m$ and $n$ are introduced as $m=N_{y}(r-1)+b$ and $n=N_{y}(i-1)+j$ for $i, r=1, \ldots, N_{x}$ and $j, b=1, \ldots, N_{y}$. Hence,

Eq. (4) can be written more succincly as

$\omega_{r b}^{2} q_{m}+\ddot{q}_{m}+\sum_{n=1}^{N} A_{n, m} \ddot{q}_{n}+\sum_{n=1}^{N} B_{n, m} q_{n}+\sum_{n=1}^{N} C_{n, m} \ddot{q}_{n}=0, \quad m=1, \ldots, N$,

where $N=N_{x} N_{y}$. For harmonic excitation Eq. (8) can be further simplified by substituting $\ddot{q}_{n}=-\omega^{2} q_{n}$. This yields

$\omega_{r b}^{2} q_{m}+\sum_{n=1}^{N} B_{n, m} q_{n}-\sum_{n=1}^{N}\left(A_{n, m}+C_{n, m}+\delta_{n m}\right) \omega^{2} q_{n}=0, \quad m=1, \ldots, N$ 
Eq. (9) can be expressed in matrix form as

$[K]\{q\}=\omega^{2}[M]\{q\}$,

where $[K]=\left[\omega^{2}\right]+[B],[M]=[A]+[C]+[I]$ which are both $(N \times N)$ matrices, $\left[\omega^{2}\right]$ is a diagonal matrix with the resonant frequencies of the unconstraint plate and $[A]$, $[B]$ and $[C]$ are $(N \times N)$ matrices given by Eqs. (5), (6) and (7), respectively. The integral expressions in Eqs. (6) and (7) must be solved numerically. The differentiation in Eq. (6) can be solved analytically using Leibniz rule. Eq. (10) represents an eigenvalue problem that can be solved numerically. The eigenvalues $\omega$ are the natural frequencies of the modified structure and the eigenvectors $\{q\}$ can be used in Eq. (3) to obtain the mode shapes of the modified structure. Full details of the theoretical approach are given in Ref. [12]. A downloadable copy of Ref. [12] is available from the Lougborough University Institutional Repository.

\subsection{Acoustical model}

The sound power radiated by a given mode can be calculated by means of the velocity spectrum of its mode shape and the mode shape can be calculated by solving the eigenvalue problem in Eq. (10) and using the determined eigenvectors with Eq. (3). The sound power radiatied by a given mode can be evaluated in the wavenumber domain using [2]

$\bar{P}_{\mu}=\frac{\rho_{0} c k_{\mu}}{8 \pi^{2}} \iint_{-\infty}^{\infty} \operatorname{Re}\left[\frac{\left|U_{\mu}\left(k_{x}, k_{y}\right)\right|^{2}}{\sqrt{k_{\mu}^{2}-k_{x}^{2}-k_{y}^{2}}}\right] \mathrm{d} k_{x} \mathrm{~d} k_{y}$,

where $U_{\mu}\left(\left(k_{x}, k_{y}\right)\right.$ is the two-dimensional velocity wavenumber spectrum of the $\mu^{\text {th }}$ mode shape, which can be calculated efficiently using a 2D Fast Fourier Transform (2D-FFT). Since only the real part of the integrant contributes to the integral equation, the limits of the integral become finite with values $-k_{\mu}$ to $k_{\mu}$, hence, numerical evaluation of the integral is possible. It is only necessary to ensure that the $2 \mathrm{D}$-FFT of 
the mode shape ranges from at least $-k_{\mu}$ to $k_{\mu}$, where $k_{\mu}=\omega_{\mu} / c$ is the acoustic wavenumber for the $\mu^{\text {th }}$ natural frequency.

\subsection{Optimisation to achieve weak acoustical radiation}

The aim of the optimisation is to impose on the structure vibration modes that radiate sound inefficiently. Hence, the objective function that needs to be minimised is given by Eq. (12). The sound power prediction made using the 2D-FFT was found to be computationally more efficient when tested against prediction formulas using the radiation matrix approach given by Ref. [13]. Since the computational cost of calculating the radiation matrix increased dramatically when the number of sampling point in the velocity profile of the plate was increased [12].

The total sound power radiated by a given number of structural modes is

$$
\bar{P}=\sum_{\mu=1}^{M} \bar{P}_{\mu}
$$

where $\bar{P}_{\mu}$ is the sound power radiated by the $\mu^{\text {th }}$ mode and $M$ is the number of structural modes. The objective function of Eq. (12) was minimised using a genetic algorithm, categorised as a global search heuristic optimiser. The methodology of genetic algorithms, inspired by evolutionary biology, is to evolve a set of candidate solutions, called the population, towards a better solution. In this case each solution, each individual in the population, is a set of data about the location and the material and geometric properties of a number of attached masses and beam-stiffeners on the plate. These data are evolved in each iteration, or generation, in order to minimise the objective function. The method is described in more detail in Ref. [12].

By inspection of Eq. (10) it can be observed that the task of the optimiser is to create an optimum modal weight vector, $\{q\}$, that will give weakly radiating mode shapes at their natural frequencies. An alternative to this would be to calculate the optimum modal weight vector from a purely acoustic optimisation procedure, as described by Naghshineh et al. in Ref. [14] and then try to adjust the structural parameters in order to obtain the desired modal weight vector. However, one of the problems with this approach is that matching the calculated modal weight vector with 
the desired modal weight vector cannot be achieved perfectly under all constraint mass and stiffener conditions. Thus, the resulting mode shapes, even though they are close to the optimum mode shapes, do not necessarily radiate acoustic energy inefficiently. Hence, the solution of the direct problem, via Eq. (10), is the approach adopted for this research.

\section{Application of point masses and line stiffeners to a vehicle body panel}

In this section the proposed optimisation design method is applied to the simplified vehicle model shown in Fig. 1. The structure is excited by two point forces in the front part of the vehicle which are assumed to be simulating the operational forces from the engine at the engine mount locations. The panel to be optimised is the left-front floor panel which can be identified by the local coordinate system symbol $z$ shown in Fig. 1. The entire floor panel consists of four identical panels separated by 5 $\mathrm{cm}$ thick rectangular beams. Each sub-panel has dimensions of $0.75 \mathrm{~m}$ by $0.55 \mathrm{~m}$. The thickness of each panel is $2.5 \mathrm{~mm}$. The material used for all the panels and the beams is steel with material properties Young's modulus $E=200 \mathrm{GPa}$ and density $\rho_{p}=7872$ $\mathrm{kg} / \mathrm{m}^{3}$.

\subsection{Identification of the boundary conditions}

Using the proposed method the panel will be conceptually 'isolated' and then its structural modes will be optimised for minimum sound radiation. The first step in the methodology is the identification of the appropriate boundary conditions that need to be applied to the isolated panel previously connected to the rest of the structure. Isolating the panel means that only the nodes and elements of the panel under consideration need to be considered whereas all other nodes and elements from the simplified vehicle FEM model can be disregarded. Thus, appropriate boundary conditions need to be applied to the nodes previously connected to the disregarded elements. Hence, translational and rotational stiffnesses are imposed onto these boundary nodes such that the isolated panel has the same vibroacoustic behaviour as that of the panel when connected to the rest of the vehicle structure. The isolated 
panel can then be optimised and should retain its optimum characteristics even when it is reattached to the rest of the vehicle structure.

Several methods have been proposed for the identification of the boundary conditions of a structure. Ahmadian and his co-researchers [15] have proposed a boundary condition identification method based upon a reduced characteristic equation of the structure. They applied this method for the determination of the translational and rotational stiffnesses at the boundaries of a beam. Pabst and Hagedorn [16] proposed a method that uses measured modal data to identify the properties of the boundary conditions of a structure. A boundary identification method using neural networks was proposed by Takahashi and Chishaki [17].

The boundary condition identification method used in this paper is similar to that reported by Zhu and Huang [18]. In Ref. [18] the vibrational response at a specific point of the structure is calculated for a given excitation force and excitation location. This data is then used to estimate the appropriate boundary conditions. For the identification of the properties of the boundaries of the floor panel of the simplified car model shown in Fig. 1, the point mobility of the panel when attached to the vehicle structure is calculated using FEM. This point mobility is calculated at a node close to the corner of the panel but not at any of the actual boundary nodes. The reason for this choice of the location is to avoid any nodal points and, hence, excite as many of the lowest order modes of the floor panel as possible. The point mobility of the isolated panel is then calculated at the same location and compared to that of the floor panel for different values of translational and rotational stiffnesses at the boundary nodes. Fig. 2 shows the modulus of the point mobility of the floor panel over the frequency range $10 \mathrm{~Hz}$ to $200 \mathrm{~Hz}$ as well as the point mobilities of the isolated panel calculated with two different sets of values for the elastic boundary conditions.

The boundary condition values used for the 'Panel elastic $\mathrm{BC} 1$ ' data shown in Fig. 2 are $10^{10} \mathrm{~N} / \mathrm{m}$ for the translational stiffness and $1000 \mathrm{Nm} / \mathrm{rad}$ for the rotational stiffness. It can be seen in Fig. 2 that the resonant frequencies of the floor panel point mobility and the resonant frequencies of the 'Panel elastic $\mathrm{BC} 1$ ' data do not exactly match. Better agreement is achieved with the 'Panel elastic BC2' data. The value for the translational stiffness for 'Panel elastic BC2' is $10^{10} \mathrm{~N} / \mathrm{m}$ and for the rotational stiffness it is $5000 \mathrm{Nm} / \mathrm{rad}$. These are boundary conditions values that can be considered close to a clamped boundary condition. Three assumptions are made 
during the methodology presented above. The first one is that the boundary conditions that need to be identified are linear. This assumption is valid for small displacements of the panel. Another assumption is that since the frequency range of interest is relatively narrow, the elastic properties of the boundaries are frequency independent. The last assumption is that boundary conditions are the same for the whole perimeter of the panel.

The point mobility of the floor panel (solid line) shown in Fig. 2 will contain the resonant frequencies due to the panel boundaries as well as some of the resonant frequencies of the whole structure. To identify an individual resonant frequency of the panel, its corresponding displacement profile at the frequency of interest can be inspected. Fig. 3a shows the displacement profile of the panel at $57 \mathrm{~Hz}$ which can be identified as the $1^{\text {st }}$ modeshape of the panel. Fig. 3b shows the displacement profile at $80 \mathrm{~Hz}$. It can be assumed that this is not a modeshape of the isolated panel, since it does not have the characteristics of an isolated panel modeshape, such as orthogonality [19]. Using this approach all of the resonant frequencies of the individual panel in the given frequency range have been identified and are shown by arrows in Fig. 2.

\subsection{Structural analysis of the automotive-type panel with added point masses and line stiffeners}

Once the appropriate boundary conditions of the isolated panel have been identified its structural modes can be optimised. For the automotive-type panel, FEM is used to calculate the modeshapes and natural frequencies of the modified panel. There are two reasons why FEM is used. The first reason is that in the analytical model of the simply-supported rectangular plate, the number of structural modes of the unmodified plate used in the analysis, which is related exponentially to the time required for the calculations, needs to be increased for more severe discontinuities. For example, heavier point masses and stiffer beams. Hence, for a given number of modes the analytical model is limited to less severe discontinuities. FEM does not suffer from this restiction. The second advantage of FEM is that the model can be extended to incorporate structures of arbitrary geometry. Hence, a mesh of any structure, not necessarily a flat plate, can be imported and optimised. 
The freely distributed code DistMesh was used for the triangular mesh generation [20]. This code was chosen because a number of fixed nodes can be defined in the geometry to be meshed. The data for the panel mesh along with the material data, the thickness of the plate, the dimensions of the cross section of the stiffeners, the weight of the masses and the translational and rotational stiffness at the boundaries are used with Nastran in order to calculate the modeshapes and the natural frequencies of the panel. The nodal displacements of each mode are then converted to nodal velocities by multiplying each value by $\mathrm{j} \omega$ in order to proceed to calculate the sound power of each mode.

\subsection{Acoustical analysis of the automotive-type panel}

The sound power radiated by each structural mode is calculated based on the method described by Cunefare and Koopmann [21]. For this method an impedance matrix is required that relates the nodal velocities to the nodal sound pressures through the equation

$p=Z u$,

where $p$ and $u$ are vectors with nodal pressures and normal velocities, respectively, and $Z$ is the impedance matrix. The impedance matrix can be derived from the influence matrices in a Boundary Element Method (BEM) analysis. In the case of a flat baffled panel the analysis can be greatly simplified by using a Rayleigh based BEM formulation. In that case the impedance matrix, for long acoustic wavelengths compared to the elements size, is given by Ref. [13] as

$Z_{i j}=\frac{j \omega \rho_{0} \exp ^{-j k R_{i j}},}{2 \pi R_{i j}}$

where $R_{i j}$ is the distance between the $i$ and $j$ nodes. The leading diagonal elements of the impedance matrix are given by $Z_{i i}=\frac{\omega \rho_{0} k}{2 \pi}$. Note, that in Ref. [13] each 
component of the matrix is multiplied by the area of each element. In the analysis presented here this is carried out later in the procedure using Eq. (17).

Based on the analysis of Cunefare and Koopmann [21] the sound power of the $n^{\text {th }}$ mode is given as:

$P_{n}=u^{T} \mathbf{B} u^{*}$,

where the superscripts $T$ and * indicate vector transpose and complex conjugate, respectively, and

$\mathbf{B}=\frac{1}{4}\left(\mathbf{A}+\mathbf{A}^{H}\right)$

where the matrix $\mathbf{A}$ can be constructed from the submatrices $\mathbf{A}_{j}$. Assuming the same interpolation functions for both the structural and acoustic elements, $\mathbf{A}_{j}$ is given by:

$\mathbf{A}_{j}=\mathbf{Z}_{j}^{T}\left(\int_{S_{j}} N N^{T} d S_{j}\right) S_{j}$

where $N$ is the vector of interpolation functions of the element $j, S_{j}$ is the area of the same element and $\mathbf{Z}_{j}$ is the submatrix of the radiation impedance matrix that yields the pressure at the nodes of element $j$.

\subsection{Optimisation of the panel design in order to achieve weak acoustical radiation}

The optimisation algorithm is based upon a genetic algorithm described in Refs. [9, 12]. Each iteration of the optimisation algorithm generates design variables related to the modification of the panel. Based upon these variables the modes of the panel are calculated. The calculated nodal displacements for all modes included in the optimisation are used to calculate the total radiated sound power radiated, which is the objective function that needs to be minimised. The sound power of each mode is calculated using Eq. (15). 
To validate the implementation of the sound power calculation presented above, the radiation efficiency of a given mode for a simply supported plate was calculated for which the analytical expression already exists [22]. Ref. [12] gives a graphical comparison of the radiation efficiency for the $(3,1)$ mode of a simplysupported plate calculated analytically and with the above method. It can be seen in Fig. 5.5 of Ref. [12] that both methods produce identical results.

The design variables for the automotive-type panel optimisation are the same as those used in Section 2 for the simply-supported rectangular panel. E.g., the coordinates of the line stiffeners, the dimensions of the cross-section of the stiffener, the coordinates of the point masses and their respective weight. The material used for the beam stiffeners is the same as that of the plate. Once the optimum parameters for the modification of the 'isolated' panel have been found they are applied to the floor panel of the simplified vehicle model. The whole structure is then excited by two point forces at the front part of the vehicle and its structural response is calculated. The radiated sound power and radiation efficiency of only the left front floor panel is then calculated using LMS Virtual Lab and the Indirect BEM.

\section{Experimental apparatus and measurement method}

In this section the experimental apparatus and the method used to measure the radiated sound power of the simply-supported panel is presented. Because of the practical difficulties in achieving the appropriate boundary conditions for the 'isolated' automotive-type panels only the simply-supported plates were tested experimentally.

There are several standardised methods for measuring the sound power radiated by a structure. Some of them are based on sound pressure measurements whereas others are based on sound intensity. Methods based on sound pressure require the measurement of sound pressure at many different points in the acoustic field of the sound source (20 for the case of a baffled source in an anechoic environment). The measurement procedure can be very tedious and is sensitive to human error especially in the determination of the measuring points. Therefore, for sound power measurements presented in this paper the sound intensity measurement method is used. 
Photographs of the baffled simply supported plate appratus are shown in Fig. 4. Fig. 4a shows the concrete baffle which has dimensions of $1.48 \mathrm{~m}$ by $1.28 \mathrm{~m}$ and a thickness $0.1 \mathrm{~m}$. At the centre of the baffle there is a steel frame in which the test plates with dimensions $0.307 \mathrm{~m}$ by $0.208 \mathrm{~m}$ can be placed. The frame has two parts, one fixed onto the baffle as can be seen in Fig. 4a and an identically sized removable part, as shown in Fig. 4b that is screwed onto the fixed part with 14 screws. The test plate is placed between the two frames. In order to simulate simply supported boundary conditions the inside of the two steel frames houses two smaller curvededged wooden frames as shown in Fig. 4b. All the edges of the plate are also sharpened in order to have a $1 \mathrm{~mm}$ long $\mathrm{V}$ shape. In this way, if the test plate has the appropriate dimensions, the wooden frame restricts the displacement at the plate's boundaries but allows for rotation.

For the data acquisition a 4 channel spectrum analyser was used. An electrodynamic exciter was used to excite the plate and its input point mobility was measured as well as the radiated sound power via sound intensity measurements. For the input mobility measurements a force transducer was attached to the end of the electro-dynamic exciter and a lightweight accelerometer was attached at the same point on the other side of the plate. For all test plates measured, the same applied force was used. The excitation signal was a swept sine signal from $1 \mathrm{~Hz}$ to $800 \mathrm{~Hz}$ with swept time of 1 second. All measurements were carried out in the anechoic chamber at Loughborough University. The sound intensity measurements were carried out using a sound intensity probe with two preamplified condenser microphones with polarisation provided by a separate power module. A schematic representation of the measurement set-up is shown in Fig. 5. Spectrum analysis was performed on the measured time signals using a 1024 point FFT, 400 frequency lines on the positive frequency side of the spectrum and frequency span from 0 to $500 \mathrm{~Hz}$.

The sound power measurements were performed using the scanning sound intensity method as described in BS EN ISO 9614:2002 [23]. The first step in this standardised methodology is the determination of the scanning surfaces that enclose the source. For these measurements the surfaces were chosen to be the surfaces of a cuboid consisting of six rectangular surfaces of which one was the surface of the source. The remaining five surfaces that enclosed the source were the scanning partial surfaces. The dimensions of the cuboid are shown in Fig. 6. The normal intensity of each partial surface was measured by placing the two microphones of the intensity 
probe perpendicular to each surface. The partial sound power for each surface is calculated by multiplying the partial normal intensity to the area of the partial surface. The total sound power is then calculated by adding the sound power of all partial surfaces. For validation purposes the scanning was performed twice for each surface using two different scanning patterns described in BS EN ISO 9614:2002 [23].

\section{Results}

\subsection{Simply-supported rectangular steel plate}

In this section the results for a steel rectangular plate with dimensions $0.307 \mathrm{~m}$ by $0.208 \mathrm{~m}$ and thickness $0.0012 \mathrm{~m}$ are presented. These dimensions were selected as being representeative of a typical vehicle body panel. The population size used in the genetic algorithm was 40 . For the evaluation of the objective function a 512 point 2DFFT was used. Since the numerical procedure used to solve the eigenvalue problem of Eq. (10) produces mode shapes of arbitrary amplitude, all the mode shapes were normalised in order to have a maximum numerical value of 1 , before the sound power was calculated. The number of modes used in the superposition was 64 .

Fig. 7 shows the predicted sound power and mean square vibrational velocity of a flat reference panel over the frequency range 0 to $500 \mathrm{~Hz}$. For the results presented in this paper the reference value for sound power levels expressed in $\mathrm{dB}$ is $10^{-12} \mathrm{~W}$. Similarly, for mean square vibrational velocity levels expressed in $\mathrm{dB}$ the reference value is $10^{-9} \mathrm{~m} / \mathrm{sec}$. The panel was excited by a point force near to the bottom left-hand corner. Five structural resonant frequencies are apparent in Fig. $7 \mathrm{~b}$. and, as expected, five corresponding peaks in the spectrum of the radiated sound power are apparent in Fig. 7a.

Figs. $8 \mathrm{a}$ and $8 \mathrm{~b}$ show the measured sound power and measured input mobility, respectively, over the frequency range from 10 to $500 \mathrm{~Hz}$. The resonant frequencies of the test plate can be identified from the peaks in the input mobility, Fig $8 \mathrm{~b}$, at approximately 115, 165, 265, 410 and $430 \mathrm{~Hz}$. Fig. 8a shows corresponding peaks in the sound power measurement, which indicates that these modes are "strongradiators". The measured sound power data are poor at low frequencies, below 100 $\mathrm{Hz}$, due to the limitations of the measuring equipment. A comparison with the corresponding predicted data shown in Fig. 7 shows that the measured resonant 
frequencies are not identical to the predicted simply-supported plate results. This indicates that the experimental set-up has not created perfect simply-supported boundary conditions. However, both sets of data demonstrate sound radiation from the resonant modes of the plate.

\section{Added point masses}

The optimisation of the plate with the mass constraints, which did not include the matrices $B$ and $C$ in Eq. (10), took about 30 minutes to compute. In Ref. [12] it was observed that the reduction in radiated sound power was significantly improved by placing the masses and stiffeners in anti-symmetric pairs. Hence, pairs of constraints were used to generate all of the following results. As a first step, the optimal location of two pairs of masses is considered. The simplest case is that of optimising one structural mode of the plate in order for it to radiate acoustic energy inefficiently. It should be noted that because the 1st structural mode does not have any nodal lines, and hence its whole surface radiates sound, it cannot be a weak radiator.

Fig. 9a shows the design of the plate optimisied for minimum sound radiation at its $2^{\text {nd }}$ mode by using 2 pairs of masses. A photograph of the plate with the attached masses fitted in the concrete baffle is shown in Fig. 9b. The masses are glued to the plate. In this figure the location of the accelerometer used to measure the input mobility of the plate can also be seen. On the other side of the plate at the same location the plate is excited by the electro-dynamic exciter.

The predicted radiated sound power and mean square vibrational velocity of the optimised plate are shown in Fig. 10. It can be seen in Fig. 10b that there is a resonant peak in the mean squared velocity at the frequency of the 2 nd mode, at approximately $100 \mathrm{~Hz}$. However, this peak at $100 \mathrm{~Hz}$ does not appear in the sound power data shown in Fig. 10a. This means that even though energy is input to the 2nd structural mode of the plate, this energy is not radiated efficiently into the acoustic medium because the way the structure vibrates in its 2 nd mode has been altered. The excitation force was located near to the corner of the plate, at location $\left(0.1 L_{x}, 0.1 L_{y}\right)$, in order to excite all the modes in the frequency range up to $500 \mathrm{~Hz}$. In Fig. 10 it can be also seen that the sound radiation of the 3rd structural mode has also been reduced even though the structure was optimised only for the 2 nd mode. 
Fig. 11 shows the measured sound power and input mobility of the plate optimised for minimum sound radiation at its $2^{\text {nd }}$ mode. It can be seen from the input mobility measurement data, Fig. $11 \mathrm{~b}$, that the $2^{\text {nd }}$ mode of the structure is at approximately $120 \mathrm{~Hz}$. The first resonant frequency is at approximately $75 \mathrm{~Hz}$ and the $3^{\text {rd }}$ resonant frequency is at approximatly $160 \mathrm{~Hz}$. Comparing these resonant peaks with the corresponding frequencies for the sound power data, Fig. 11a, indicates that the $2^{\text {nd }}$ mode is a far less significant radiator of sound than the $1^{\text {st }}$ or $3^{\text {rd }}$ modes. Hence, even though the $2^{\text {nd }}$ mode carries vibrational energy, this energy is not radiated as sound into the acoustic medium.

\section{Added line stiffeners and point masses}

The stiffeners have a rectangular cross section, with a width of $0.005 \mathrm{~m}$, which was kept constant while the height of the beam was optimised. A drawback of adding the stiffeners to the optimisation procedure is that the integral expressions in Eq. (10) need to be computed numerically in every iteration, which increases the computation time. Also the convergence of the series summation in Eq. (3) becomes slower for stiffeners placed in an arbitrary orientation. The number of modes used in the superposition for this case was 256. For the numerical integration 30 Gauss points were used. The optimisation took around four hours to compute, which was considerably longer than for the case where only added masses where considered.

Fig. 12a shows the optimal location and weight of the contraints applied to the plate to minimise sound radiation from the $2 \mathrm{nd}, 3 \mathrm{rd}, 4$ th and 5 th structural modes using two pairs of masses and one pair of stiffeners placed anti-symmetrically. The force excitation was placed at the point with coordinates $(0.187 \mathrm{~m}, 0.03 \mathrm{~m})$ in order to excite all the modes in the frequency range 10 to $500 \mathrm{~Hz}$. It can be seen in Fig. 13a that the addition of stiffener constraints has achieved sound reduction over a wide frequency range with the sound radiation from the modes targeted by the optimisation being reduced. As a result the radiated sound power shown in Fig. 13a appears smoother against frequency and is without the large amplitude peaks at the corresponding structural resonance frequencies even though the mean squared vibrational velocity shown in Fig 13b exhibits this resonant behaviour. 
Fig. 12b shows a photgraph of the plate with the optimum modifications mounted in the concrete baffle. The excitation point is at the same location $(0.187 \mathrm{~m}$, $0.03 \mathrm{~m}$ ) as used for the predicted results shown in Fig. 13. Fig. 14 shows the measured sound power and input mobility. The input mobility data shown in Fig. 14b indicate that the $2^{\text {nd }}$ to $5^{\text {th }}$ resonant peaks are at approximately 140, 250, 330400 and $440 \mathrm{~Hz}$, respectively. It can be seen in Fig 14a, that with the exception of the $2^{\text {nd }}$ mode, modes 2-5 have weak acoustic radiation characteristics.

Despite the difficulty in obtaining perfect simply supported boundary conditions for the experimental plates, the measurement results generally confirm the numerical predictions for minimum sound radiation. However, one potential source of discrepancy between the measured and predicted results is the presence of solid bodies, i.e. the attached masses and stiffeners, in the acoustic field of the experimental plates. In the numerical predictions it was assumed that the masses and stiffeners did not interfere with the acoustic field since they were considered as a part of the flat plate. In practice, the attached bodies may create acoustic scattering of the energy radiated by the plate.

\subsection{Automotive-type panel}

In this section results are presented for the optimisation of the floor panel of the simplified vehicle model shown in Fig. 1. The upper frequency of interest is 200 $\mathrm{Hz}$ and the unmodified panel has five modes in the frequency range from $10 \mathrm{~Hz}$ to $200 \mathrm{~Hz}$. Two line stiffeners and four point masses have been used to optimise the 2nd to 6th modes of the panel. The upper weight limit for each point mass in the optimisation was $0.5 \mathrm{~kg}$ whereas the upper limit used for the dimensions of the rectangular cross section of each stiffener was $0.02 \mathrm{~m}$.

The resulting geometry of the modifications on the panel can be seen in Fig. 15. The total added mass to the panel from the point masses is $1.5 \mathrm{~kg}$, which is a $42 \%$ increase in its total weight. Figure 16a shows a comparison of the radiated sound power of the unmodified floor panel and the sound power of the panel with the optimum modifications. The $2^{\text {nd }}$ to $6^{\text {th }}$ modes of the isolated modified panel were calculated to be at 78, 103,122, 131 and $155 \mathrm{~Hz}$, respectively. There are many resonant peaks shown in Fig 16a that do not correspond to the resonances of the 
panel. These are due to the global modes of the car structure. From Fig. 16a it is difficult to determine whether a significant reduction in the sound power has been achieved for frequencies at or around the optimised modes of the panel. Thus, the radiation efficiency of the panels has also been calculated and is shown, in logarithmic scale, in Fig. 16b. It can be seen that a significant reduction has been achieved for frequencies at and around the optimised modes. Overall, the difference between the linear $\mathrm{dB}$ average of the sound power of the modified and unmodified panels over the frequency range 10 to $200 \mathrm{~Hz}$ shows a small increase of $1.1 \mathrm{~dB}$ for the modified panel. A small decrease of $0.3 \mathrm{~dB}$ was observed in the average radiation efficiency of the modified panel for the same frequency range. Note that in this frequency range there are also modes of the panel that were not included in the optimisation. In particular, this may explain the relative increase in the radiated sound power and radiation efficiency above $155 \mathrm{~Hz}$, which is the frequency of the highest optimised mode.

\section{Summary and conclusions}

A method to optimise the modes of a simply-supported rectangular plate and an automotive-type panel in order to achieve weak radiation of the acoustic energy has been presented. This was achieved by optimising the location and weight of point masses and line stiffeners added to the structure. Firstly, a theoretical structural model for a simply-supported plate with added point masses and line stiffeners at arbitrary locations and orientations was developed. This model was then used with a genetic algorithm in order to optimise the location of the constraints so as to mininise the sound power radiated by one or more targeted modes at their respective natural frequencies. The optimised plate designs were then numerically modelled using finite elements and boundary elements in order to make a prediction of the radiated sound over a wide frequency range. The optimised plate designs were tested experimentally in an anechoic chamber by mounting them within a specially designed concrete baffle.

The predicted and measured results showed that, by imposing weakly radiating modes on the plate, a combination of masses and stiffeners can be an effective noise control measure. Thus, the radiated sound power is smoother over the target frequency range and does not contain the high amplitude peaks at the resonant 
frequencies of the structure even though these resonant peaks appear relatively pronounced in the corresponding mean squared vibrational velocity response of the structure.

The proposed method was then applied to a simplified vehicle model. A floor panel of the vehicle model was conceptually isolated from the rest of the vehicle and its structural modes were optimised for minimum sound radiation using a combination of point masses and line stiffeners. Predicted results showed a reduction in the radiation efficiency of the in-situ panel at the frequencies of the modes of the isolated modified panel. However, over the entire frequency range analysed the optimised panel design produced a small increase in the total radiated sound power. Thus, for a built-up structure the global modes of the whole structure may still generate significant sound even though the sound from the target modes of the 'isolated' panel has been reduced.

One advantage of the proposed method is a reduction in the time required to optimise the design of a built-up vehicle structure. Since the panel under consideration is isolated and optimised independently, when the vehicle structure or its parts are redesigned, the optimised panel will still retain its optimum acoustic characteristics. Another advantage of this method is that the optimum panel design does not depend upon a knowledge of the excitation forces. Hence, this procedure can be used at an early stage of the vehicle design process before the structural design has been finalised and when the exact form of the excitation is unknown.

\section{References}

[1] Marburg, S. Developments in structural-acoustic optimization for passive noise control. Arch. Comput. Meth. Eng., 9:291-370, 2002.

[2] Christensen, S. T., Sorokin, S. V., Olhoff, N. On analysis and optimization in structural acoustics - Part I: Problem formulation and solution techniques. Struct. Optim., 16:83-95, 1998.

[3] Lamancusa, J. S. Numerical optimization techniques for structural-acoustic design of rectangular Panels. Comput. and Struct., 48:661-675, 1993.

[4] Marburg, S. Efficient optimization of a noise transfer function by modification of a shell structure geometry - Part I: Theory. Struct. Multidisc. Optim., 24:51-59, 2002. 
[5] Hambric, S. A. Approximation techniques for broad-band acoustic radiated noise design optimization problem. J. Vib. Acoust., 117:136-144, 1995.

[6] Koopmann, G. H. and Fahnline, J. B. Designing quiet structures, a sound power minimization approach. Academic Press, California, 1997.

[7] Wodtke, H. W. and Koopmann, G. H. Quitening plate modes with optimally sized point masses - a volume velocity approach. Proc. ASME Des. Eng. Tech. Conf., 647654, 1995.

[8] Pierre Jr., R. L. St. and Koopmann, G. H. A design method for minimizing the sound power radiated from plates by adding optimally sized, discrete masses. J. Vib. Acoust., 117:243-251, 1995.

[9] Kumar, G., Walsh S. J., Krylov V. V. Structural-acoustic behaviour of automotive-type panels with dome-shaped indentations. App. Acoust. 74:897-908, 2013.

[10] Weissenburger, J. T. Effect of local modification on the vibration characteristics of linear systems. J. Appl. Mech., 35:327-332, 1968.

[11] $\mathrm{Wu}$, J. S. and Luo, S. S. Use of the analytical-and-numerical-combined method in the free vibration analysis of a rectangular plate with any number of point masses and translational springs. J. Sound Vib., 200:179-194, 1997.

[12] Rousounelos, A. Reduction of sound radiation from automotive-type panels. $\mathrm{PhD}$ Thesis, Loughborough University, 2010.

[13] Fahy, F. and Gardonio, P. Sound and structural vibration. Radiation, Transmission and Response, $2^{\text {nd }}$ Ed., Academic Press, London, 2007.

[14] Naghshineh, K., Koopmann, G. H. and Belegundu, A. D. Material tailoring of structures to achieve a minimum radiation condition. J. Acoust. Soc. Am., 92:841$855,1992$.

[15] Ahmadian, H., Mottershead, J. E., Friswell, M. I. Boundary condition identification by solving characteristic equations. J. Sound and Vib., 247:755-763, 2001.

[16] Pabst, U. and Hagedorn, P. Identification of boundary conditions as a part of model correction. J. Sound and Vib., 182:565-575, 1995.

[17] Takahashi, K. and Chishaki, T. Free vibrations of plate structures with intermediate frames. J. Sound and Vib., 61:79-99, 1978.

[18] Zhu, J. and Huang, L. Estimation of boundary parameters of plate structures. $J$. Sound and Vib., 179:455-461, 1995. 
[19] Junger, M. C. and Feit, D. Sound, structures and their interaction. Acoust. Soc. of Am., New York, 1993.

[20] MSC Software. MSC.Nastran 2004 quick reference guide. 2004.

[21] Cunefare, K. A. and Koopman, G. H. Global optimum active noise control: surface and far-field effects. J. Acoust. Soc. Am., 90:365-373, 1991.

[22] Wallace, C. E. Radiation resistance of a rectangular panel. J. Acoust. Soc. Am., 51:946-952, 1972.

[23] BS EN ISO 9614:2002. Acoustics - determination of sound power levels of noise sources using sound intensity - part 3 precision methods for measurement by scanning. CEN, 2002. 


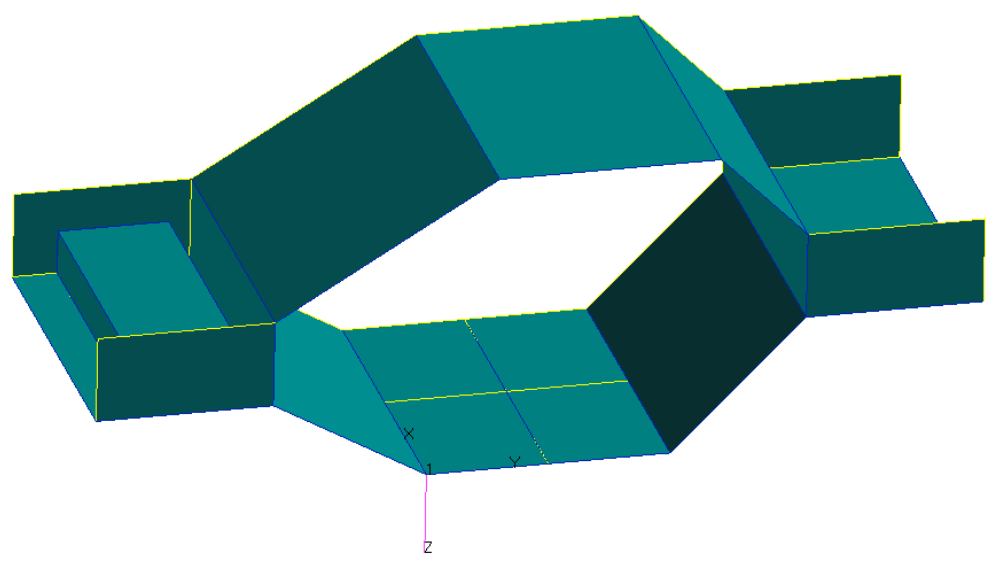

Fig. 1. Simplified vehicle model used when applying the optimisation design method to an automotive-type panel. 


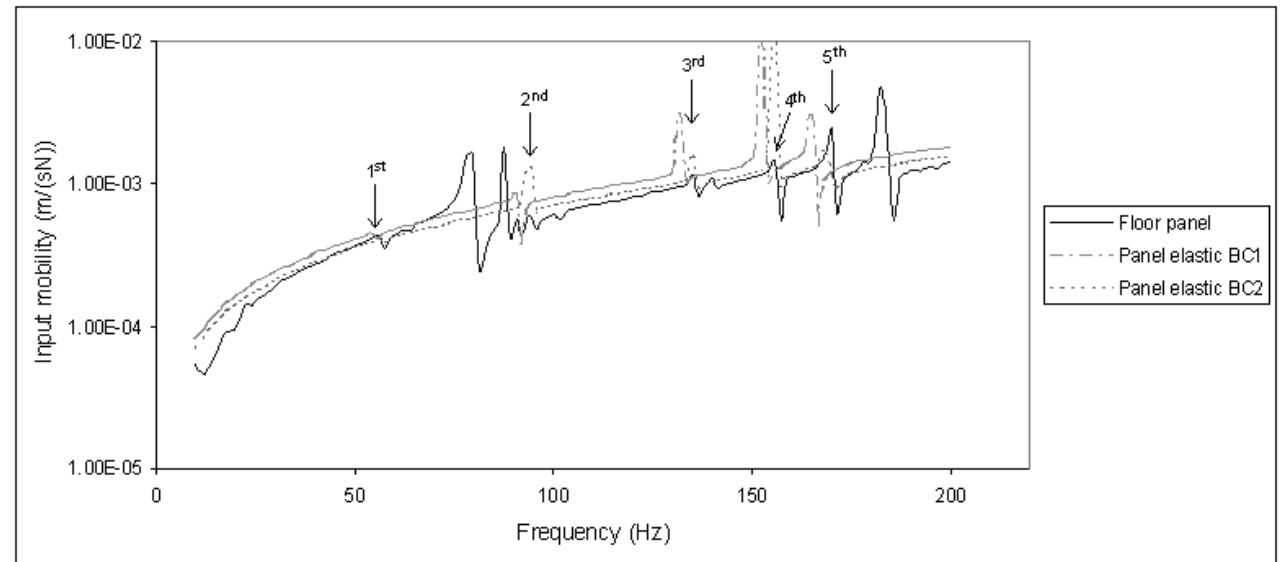

Fig. 2. Comparison of the modulus of the point mobility of the floor panel of the vehicle with the isolated panel calculated with two different sets of stiffness values. 
(a) Displacement profile at $57 \mathrm{~Hz}$

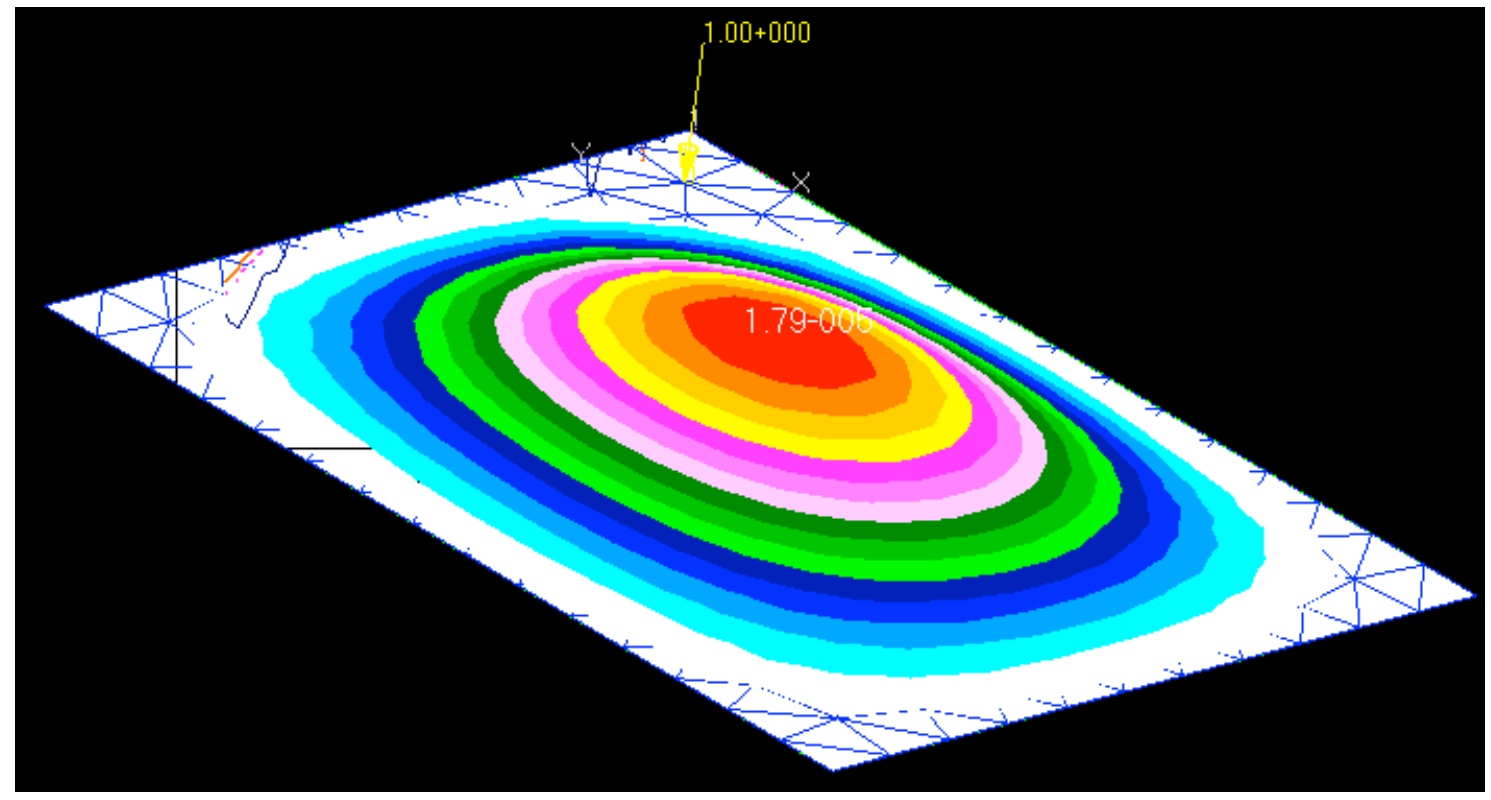

(b) Displacement profile at $80 \mathrm{~Hz}$

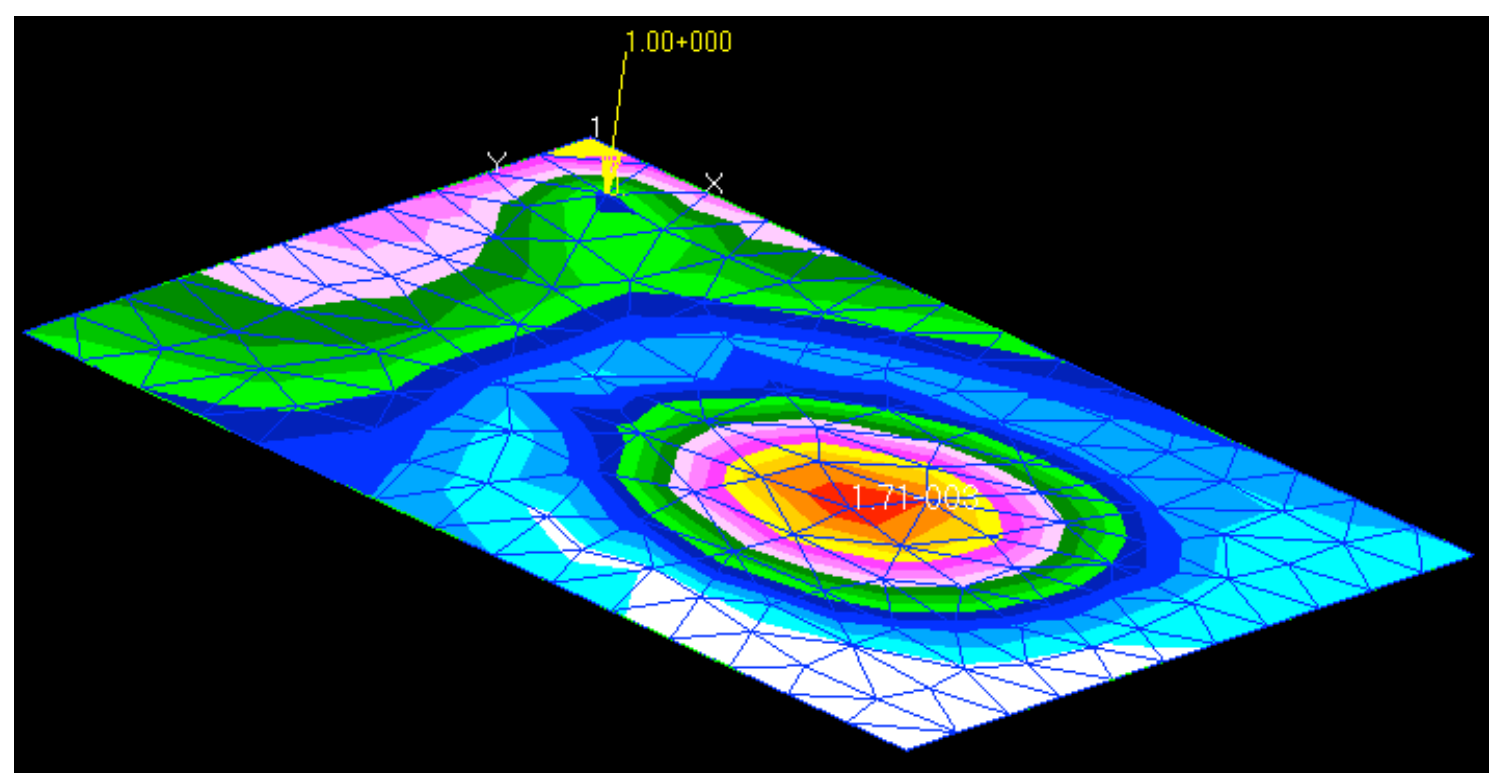

Fig. 3. Displacement profile of the panel at two different resonant frequencies illustrating: (a) a mode shape due to the individual panel boundaries; (b) a mode shape due to the complete vehicle. 
(a) Concrete baffle

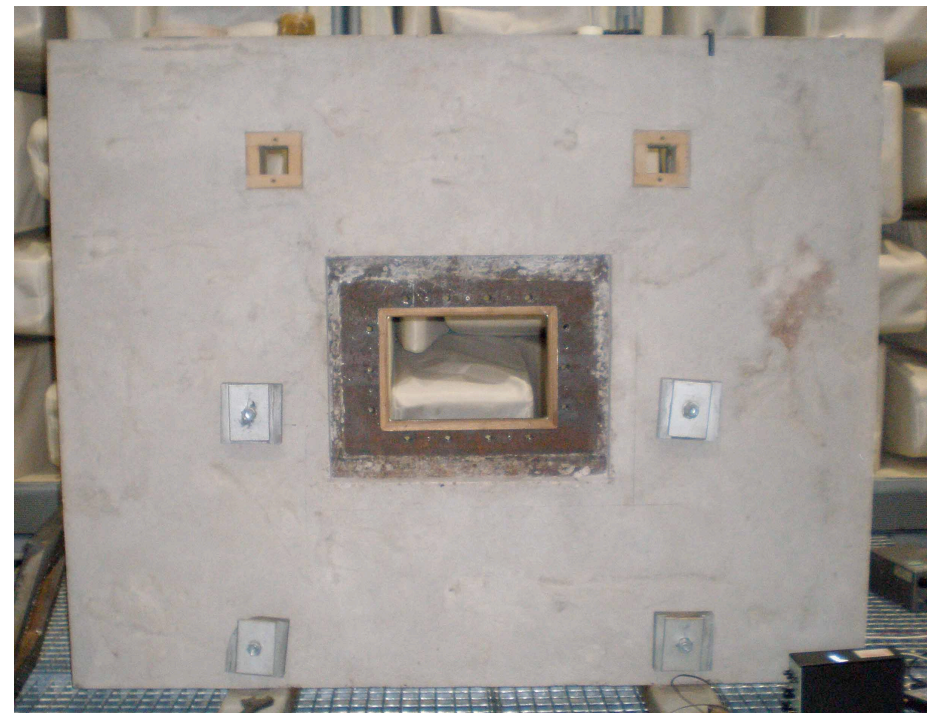

(b) Steel frame

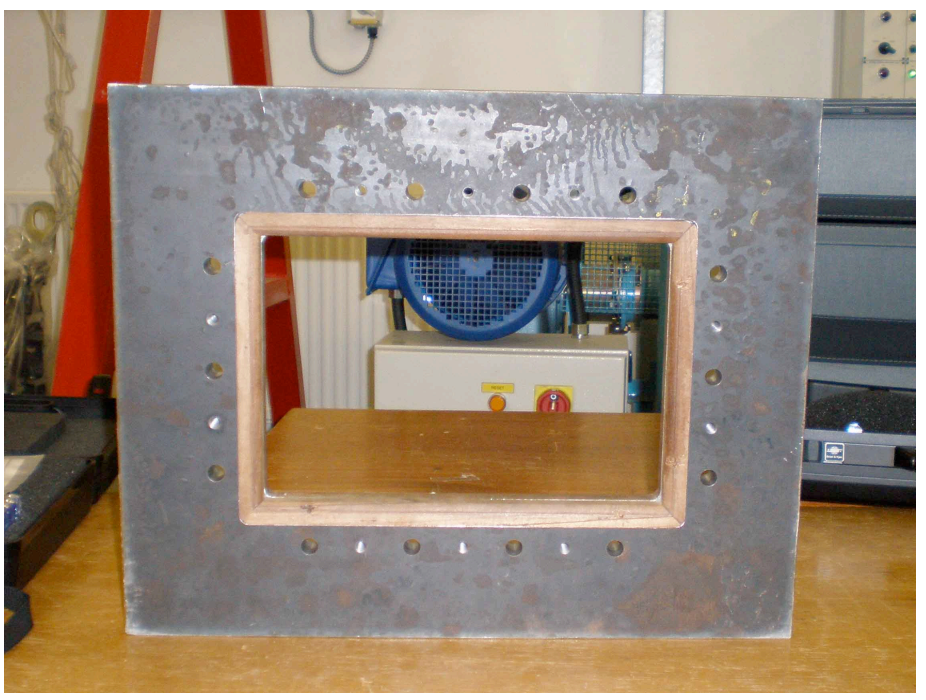

Fig. 4. Photographs of the baffled plate apparatus: (a) concrete baffle; and (b) closeup of the steel frame in which the test plate is mounted. 


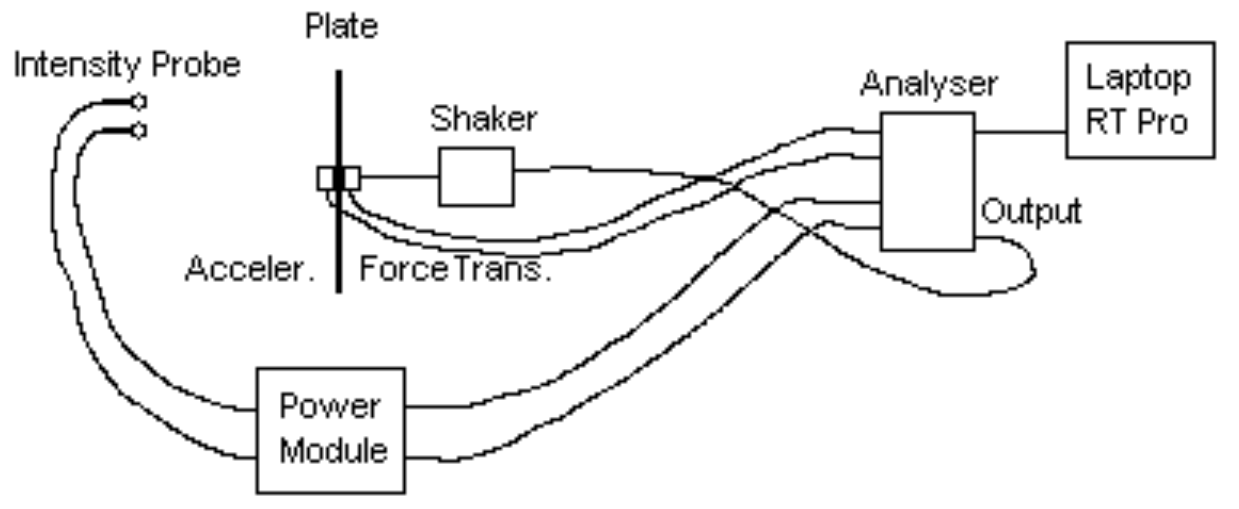

Fig. 5. Schematic representation of the measurement equipment set-up. 


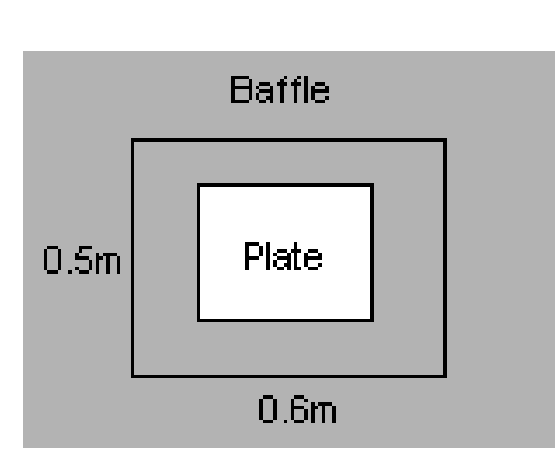

Perpendicular View

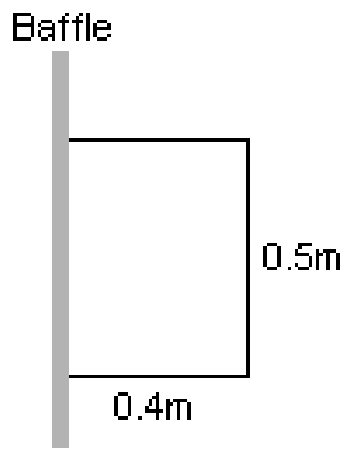

Side View

Fig. 6. Schematic representation of the cuboid that determines the partial surfaces of the scanning sound intensity measurements. 
(a) Radiated sound power

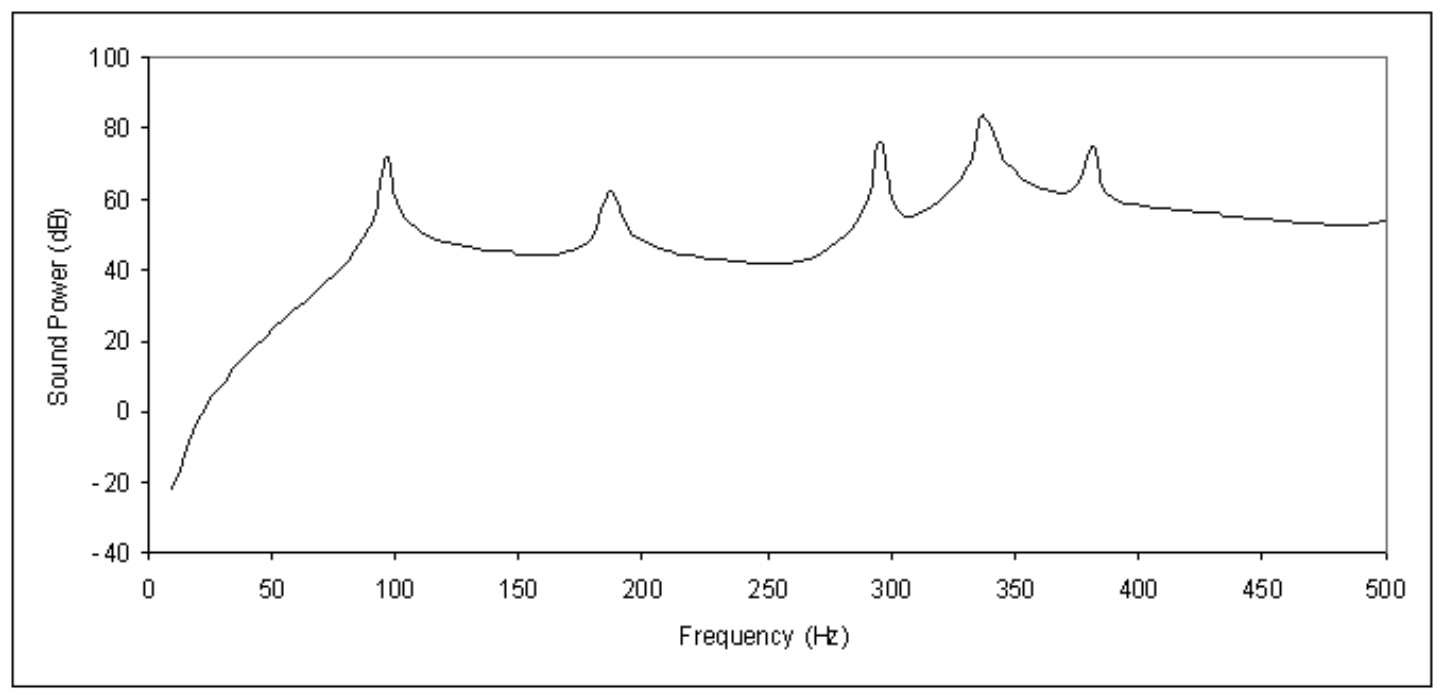

(b) Mean squared vibrational velocity

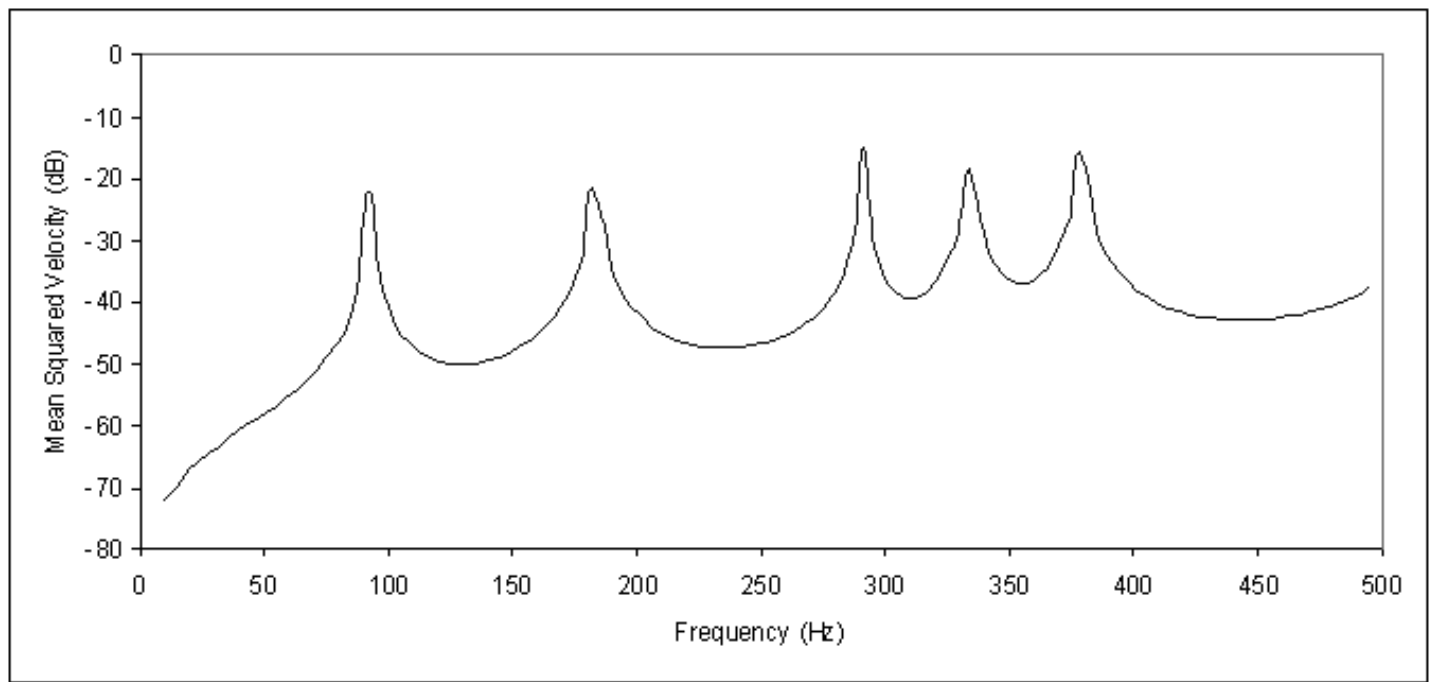

Fig. 7. Predicted results for the simply-supported flat reference panel: (a) radiated sound power; (b) mean squared vibrational velocity. 
(a) Radiated sound power

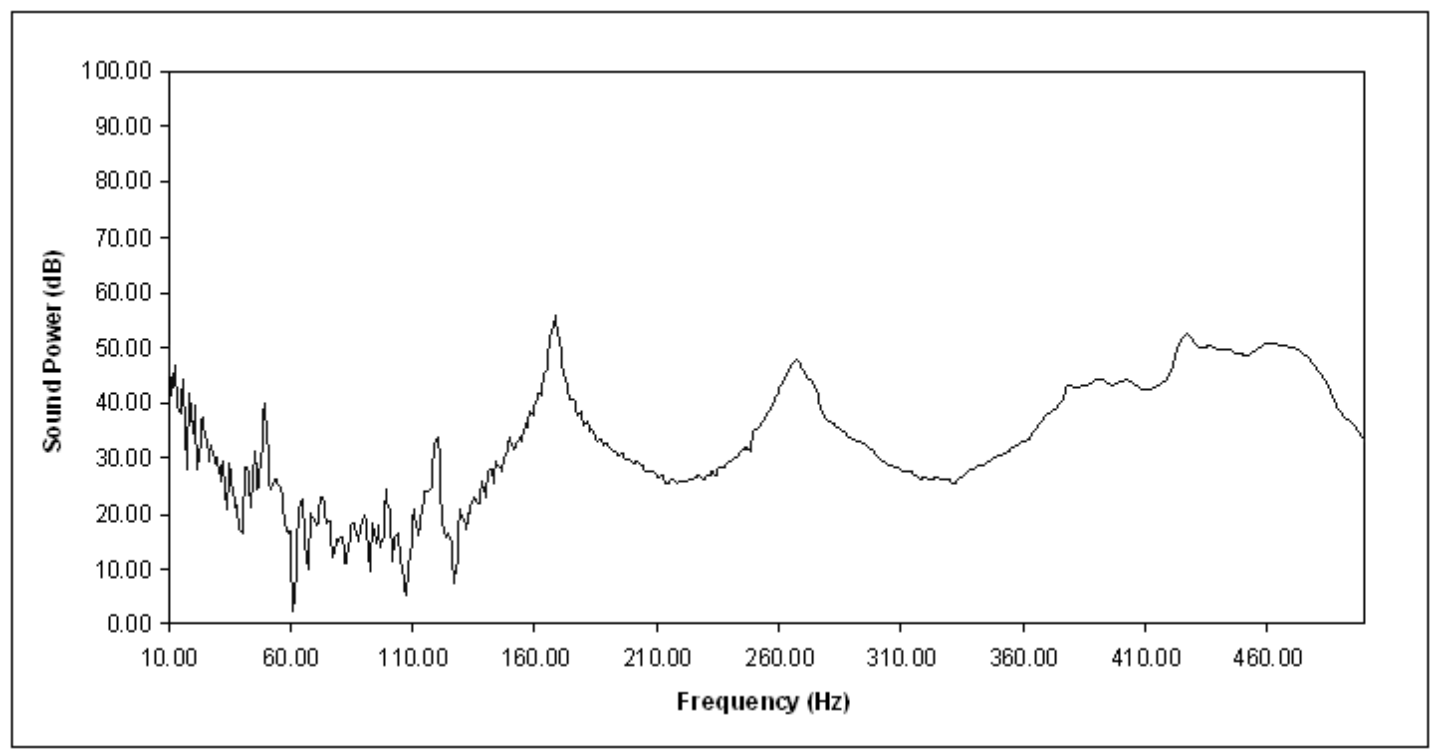

(b) Input mobility

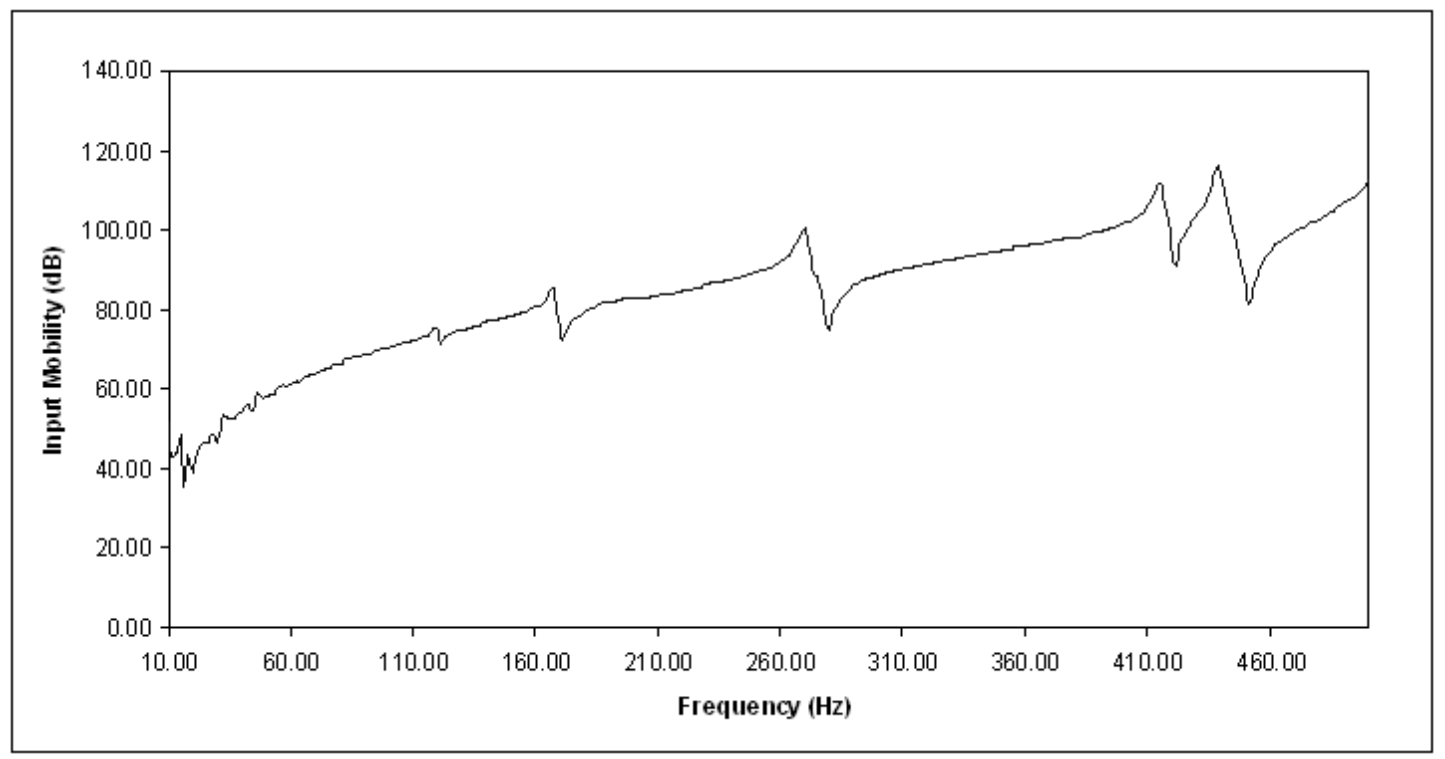

Fig. 8. Measured results for the simply-supported flat reference panel: (a) radiated sound power; (b) input mobility. 
(a) Theoretical optimum design

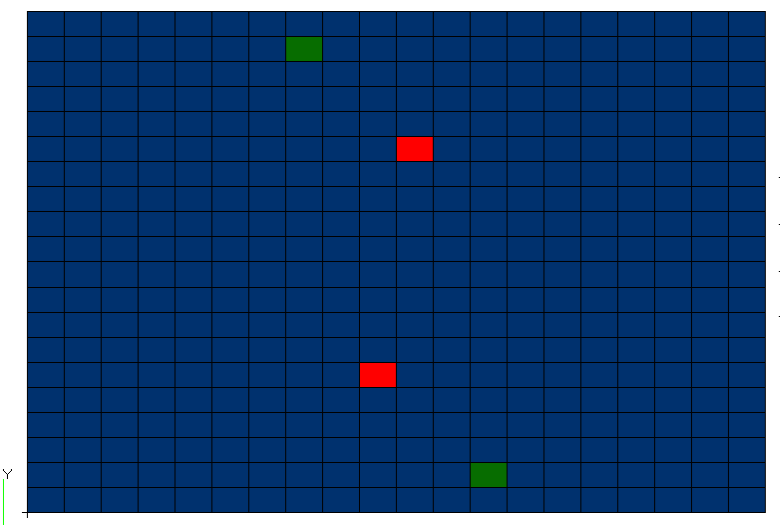

\begin{tabular}{l|l|l|l} 
& $\boldsymbol{x}(\boldsymbol{m})$ & $\boldsymbol{y}(\boldsymbol{m})$ & $\boldsymbol{k g}$ \\
\hline Mass 1 & 0.153 & 0.062 & 0.36 \\
\hline Mass 2 $\square$ & 0.123 & 0.198 & 0.12 \\
\hline Mass 3 $\square$ & 0.169 & 0.156 & 0.36 \\
\hline Mass 4 $\square$ & 0.200 & 0.021 & 0.12
\end{tabular}

(b) Experimental plate

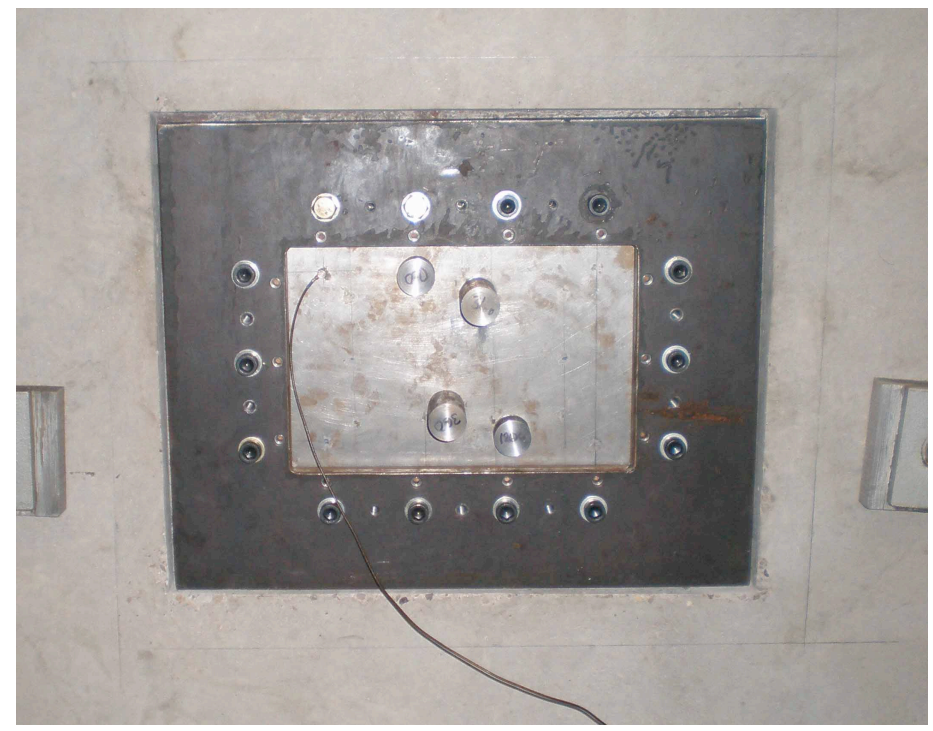

Fig. 9. Optimum location and weight of the two pairs of point masses used in order to minimise the sound radiation from the $2^{\text {nd }}$ structural mode of the simply-supported rectangular plate: (a) theoretical optimum location and weight of the two pairs of masses; and (b) photograph of the experimental plate. 
(a) Radiated sound power

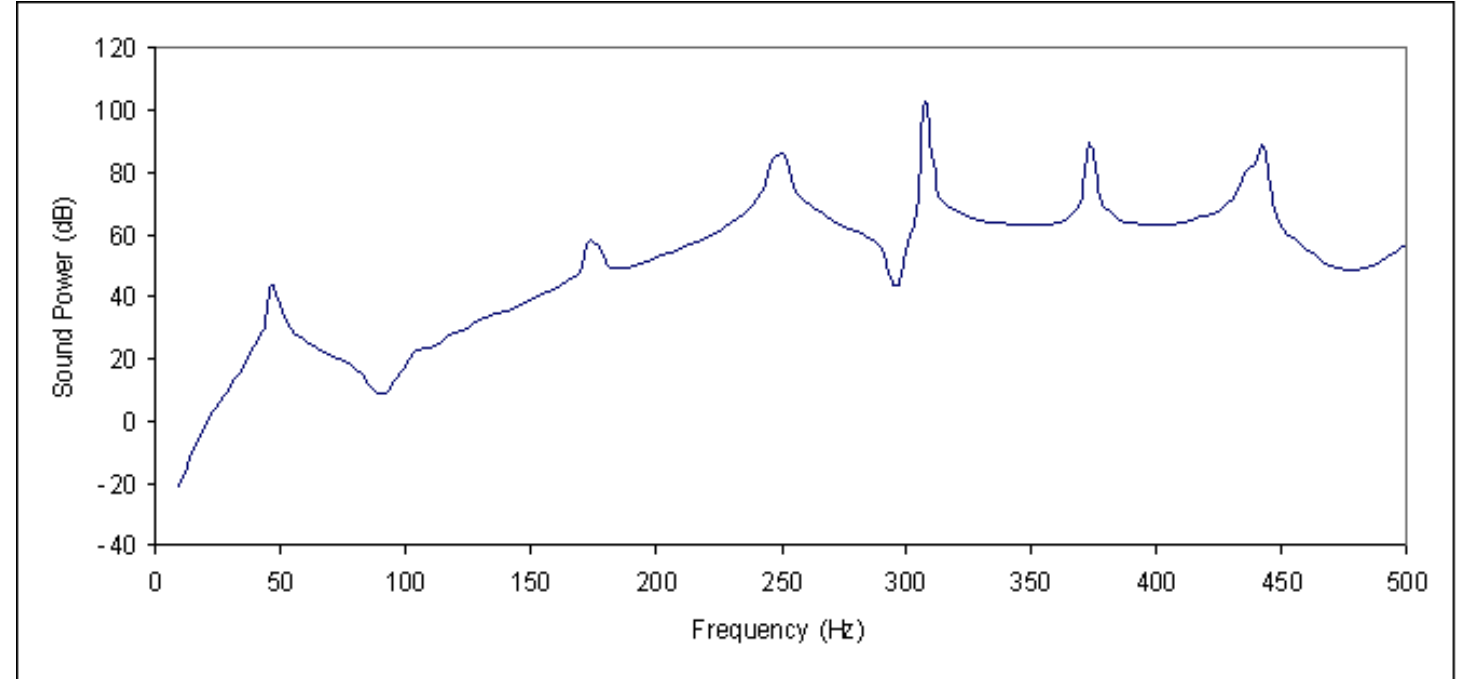

(b) Mean squared vibrational velocity

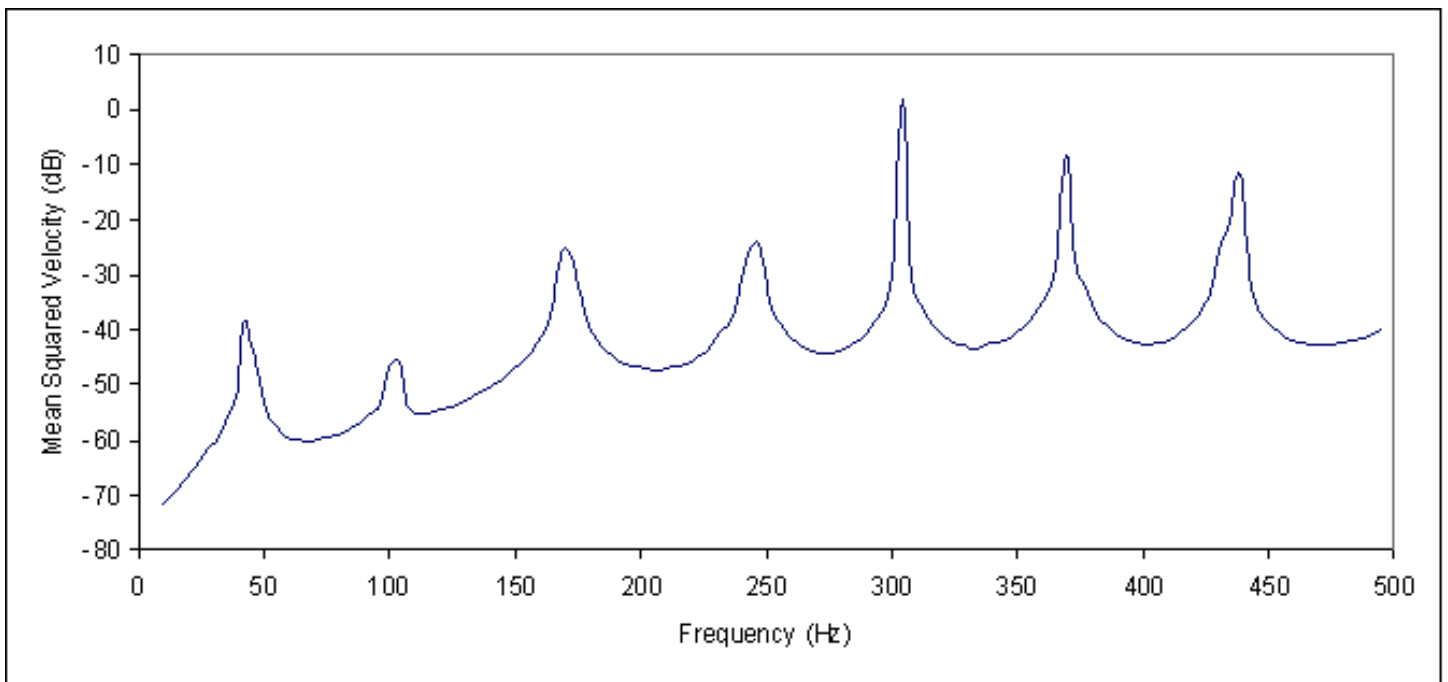

Fig. 10. Predicted result of optimising the $2^{\text {nd }}$ structural mode of the simply-supported rectangular plate for minimum sound radiation using two pairs of point masses: (a) radiated sound power; (b) mean squared vibrational velocity. 
(a) Radiated sound power

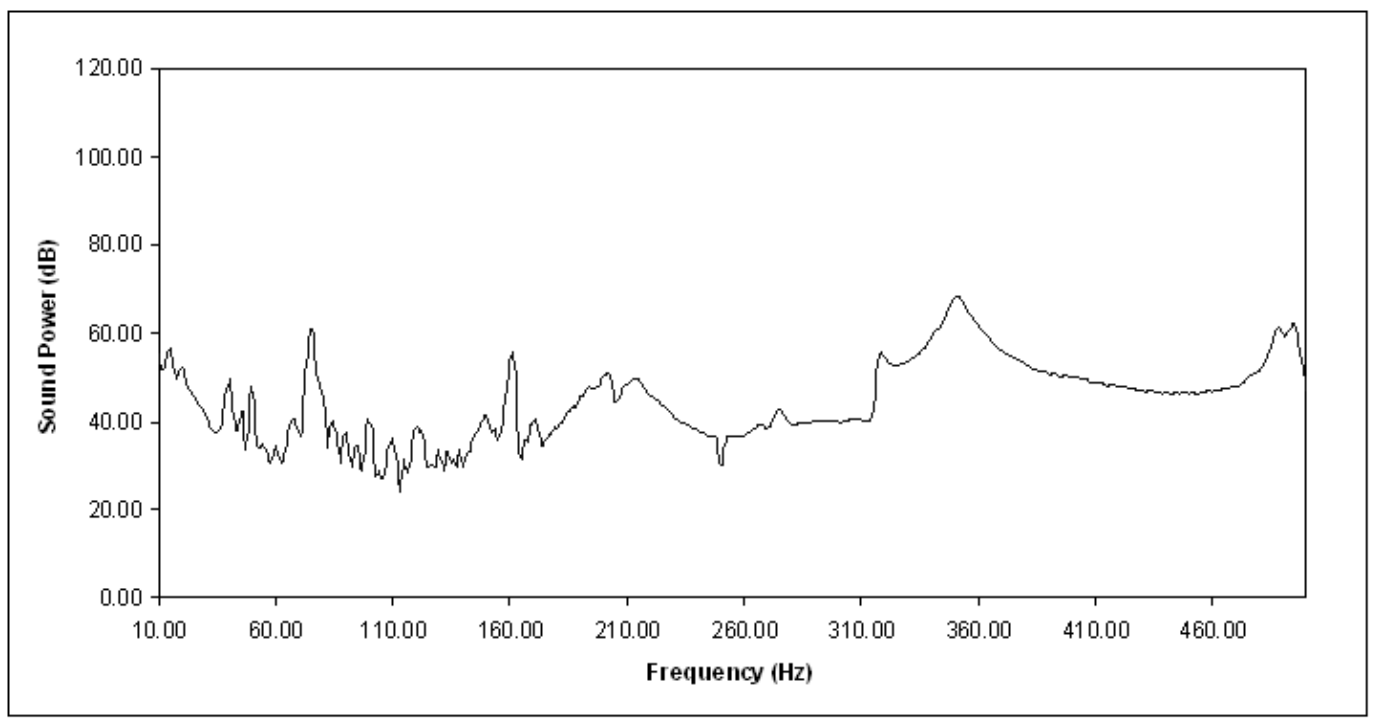

(b) Input mobility

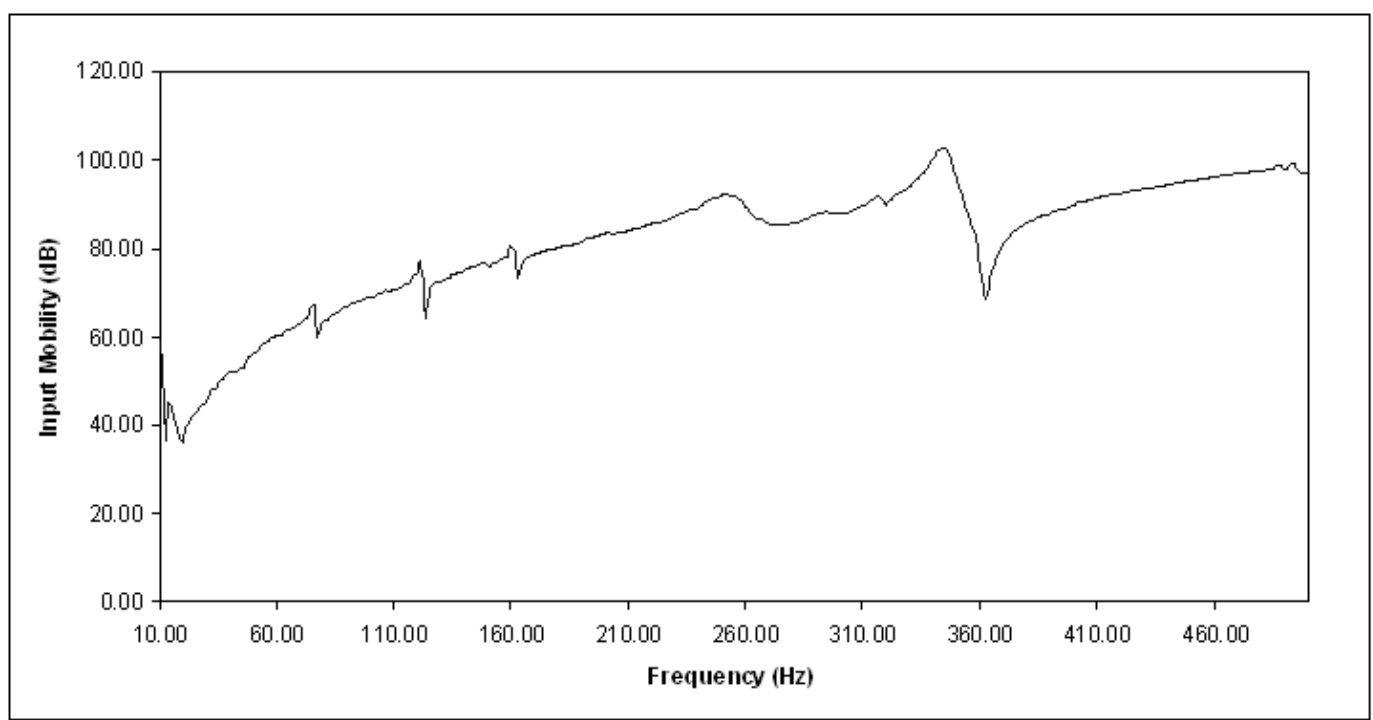

Fig. 11. Measured result of optimising the $2^{\text {nd }}$ structural mode (at approximately 120 $\mathrm{Hz}$ ) of the simply-supported rectangular plate for minimum sound radiation using two pairs of point masses: (a) radiated sound power; (b) input mobility. 
(a) Radiated sound power

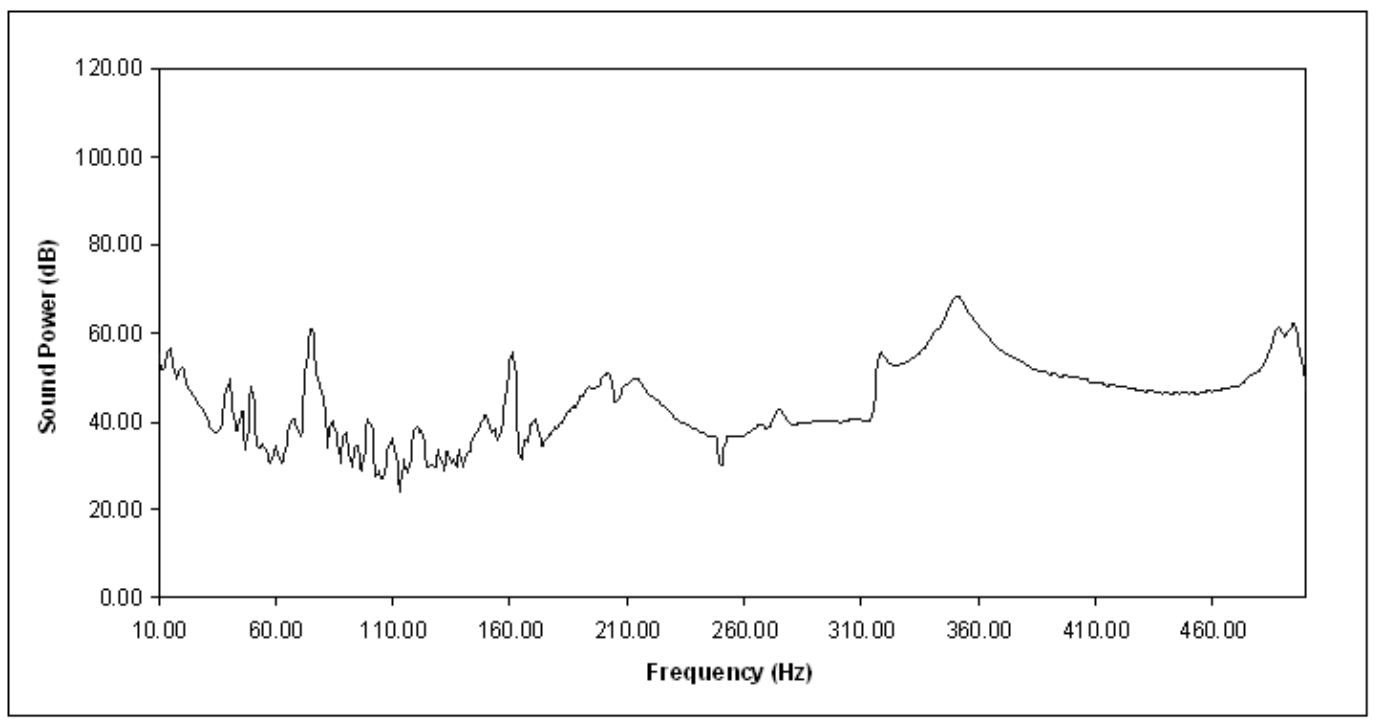

(b) Input mobility

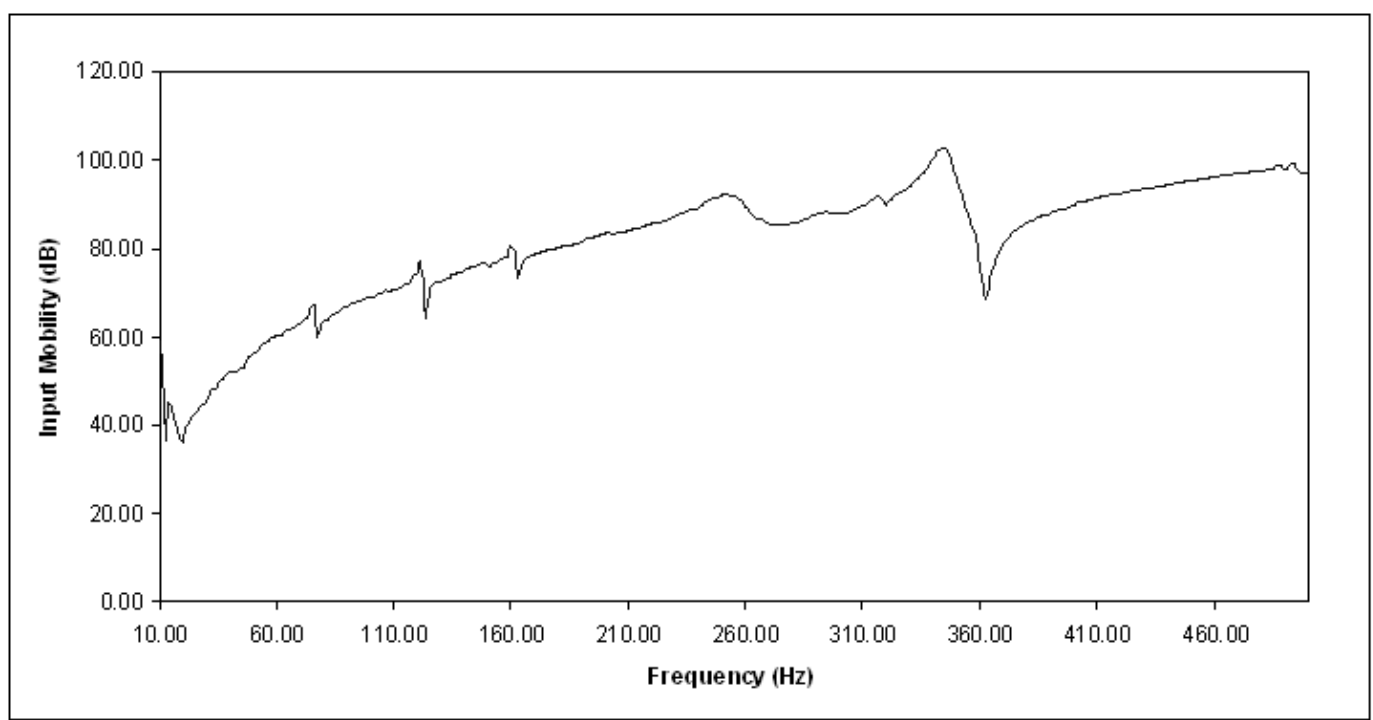

Fig. 11. Measured result of optimising the $2^{\text {nd }}$ structural mode (at approximately 120 $\mathrm{Hz}$ ) of the simply-supported rectangular plate for minimum sound radiation using two pairs of point masses: (a) radiated sound power; (b) input mobility. 
(a) Theoretical optimum design

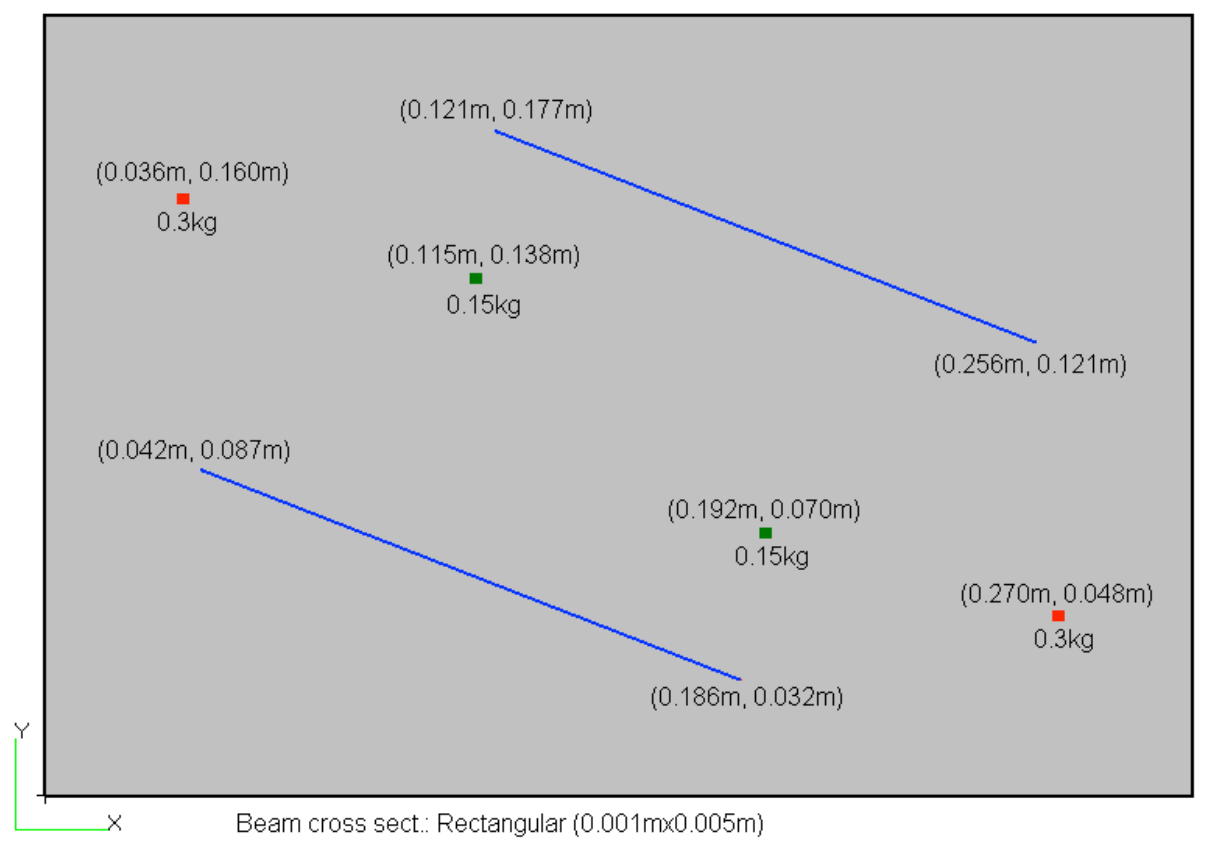

(b) Experimental plate

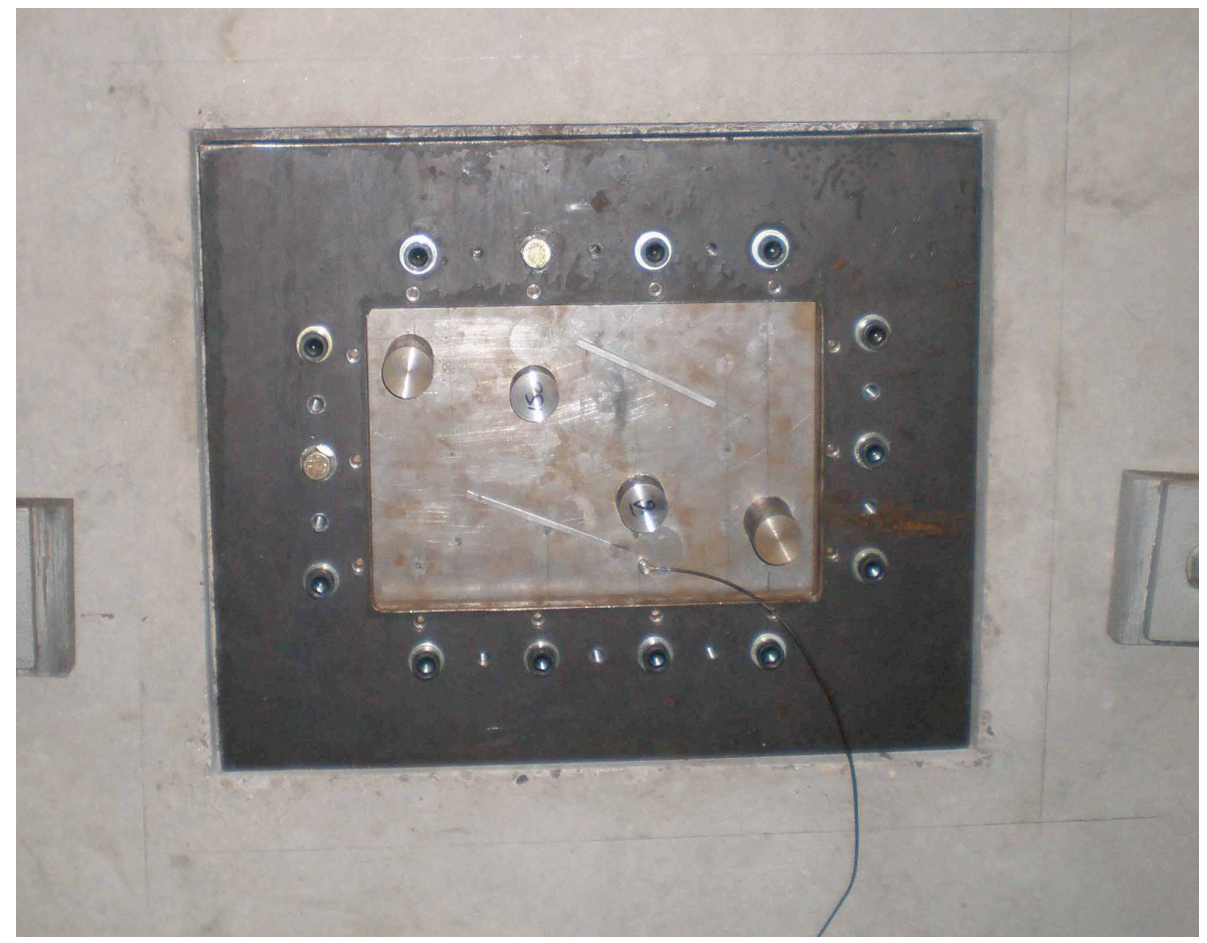

Fig. 12. Optimum location and weight of the two pairs of point masses and one pair of stiffeners used in order to minimise the sound radiation from the $2^{\text {nd }}, 3^{\text {rd }}, 4^{\text {th }}$ and $5^{\text {th }}$ structural modes of the simply-supported rectangular plate: (a) theoretical optimum location and weight; and (b) photograph of the experimental plate. 
(a) Radiated sound power

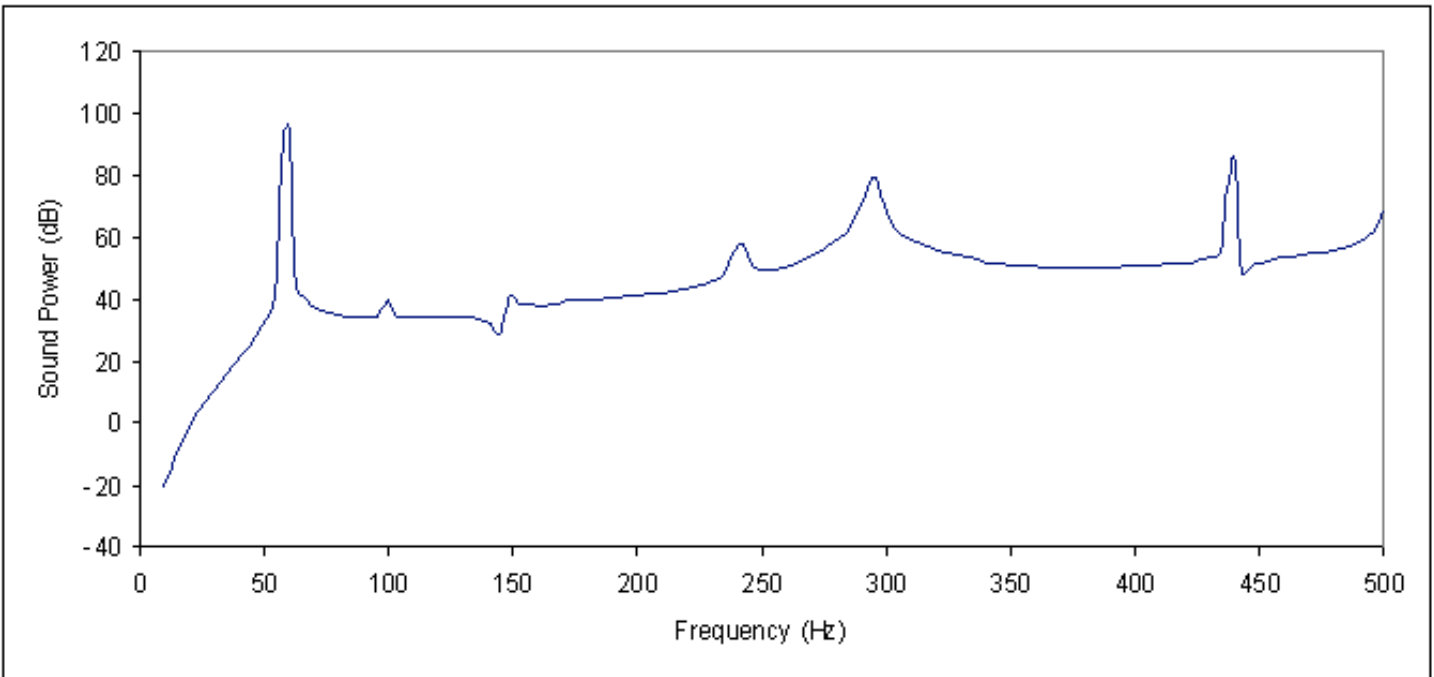

(b) Mean squared vibrational velocity

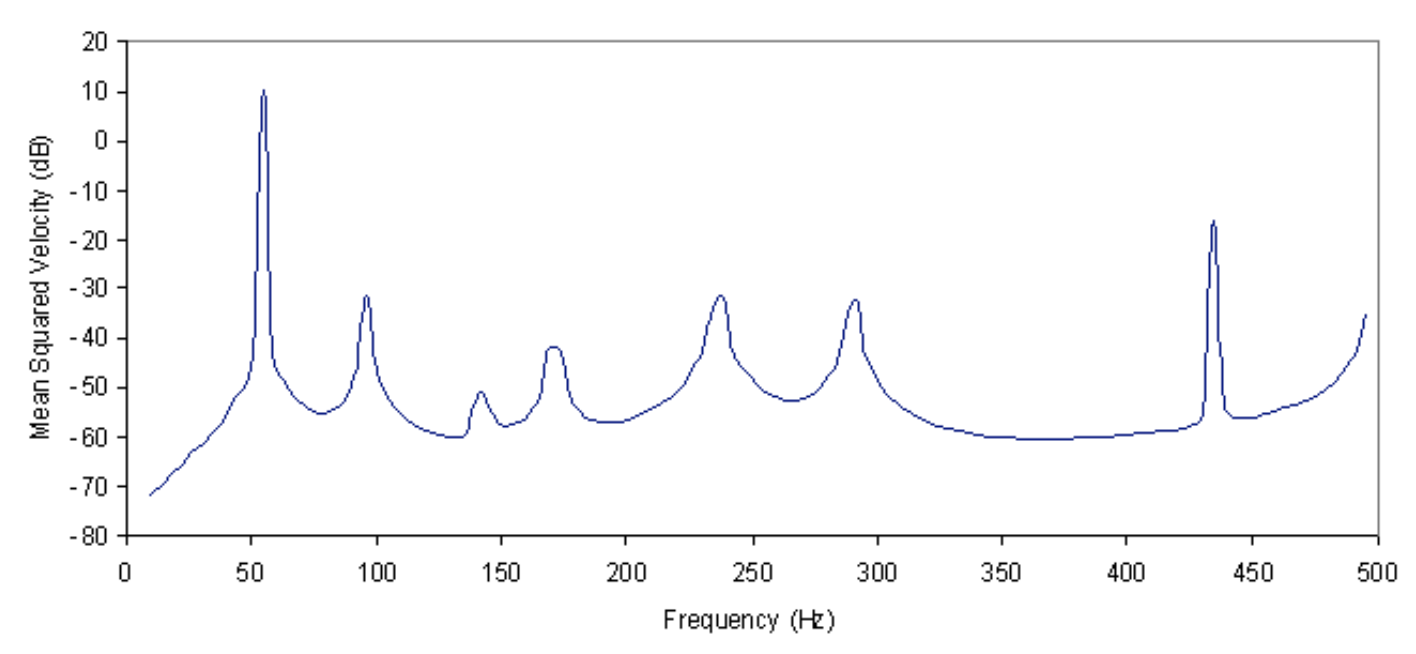

Fig. 13. Predicted result of optimising the $2^{\text {nd }}, 3^{\text {rd }}, 4^{\text {th }}$ and the $5^{\text {th }}$ structural modes of the simply-supported rectangular plate for minimum sound radiation using two pairs of point masses and one pair of line stiffeners: (a) radiated sound power; (b) mean squared vibrational velocity. 
(a) Radiated sound power

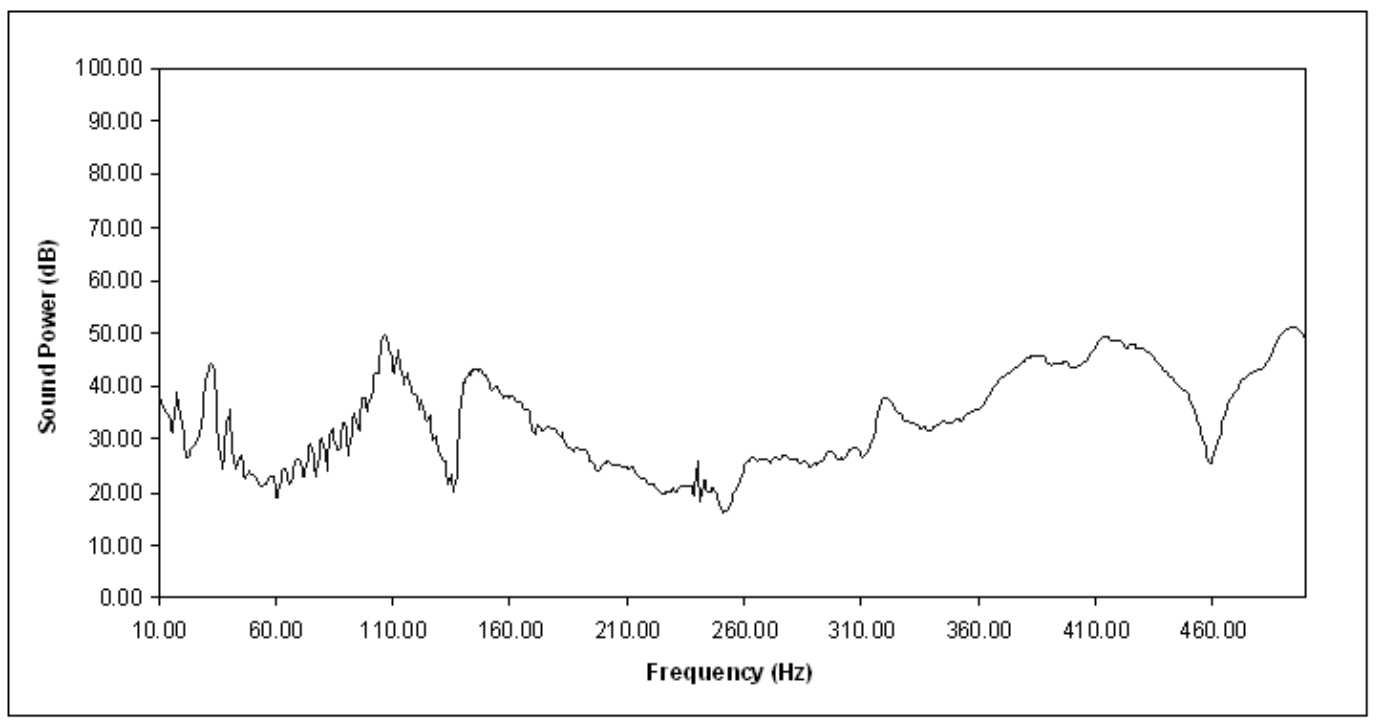

(b) Input mobility

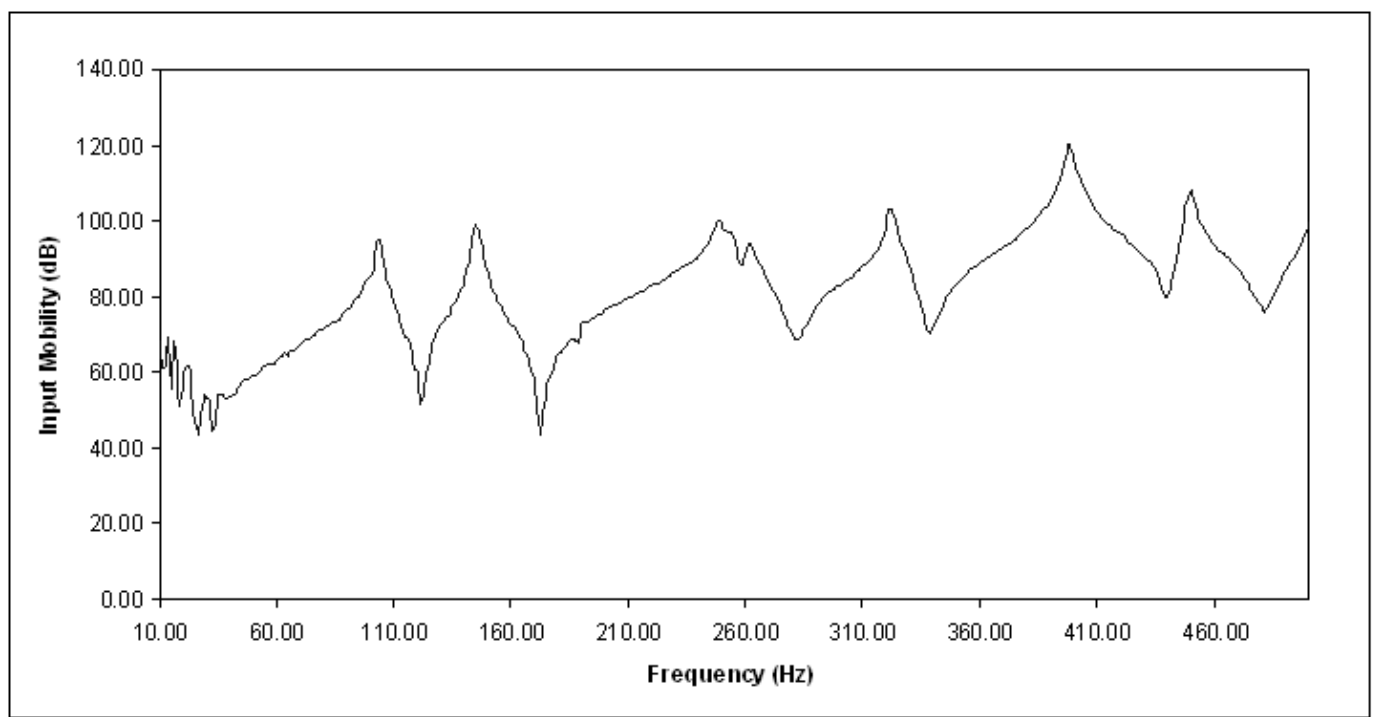

Fig. 14. Measured result of optimising the $2^{\text {nd }}, 3^{\text {rd }}, 4^{\text {th }}$ and the $5^{\text {th }}$ structural modes of the simply-supported rectangular plate for minimum sound radiation using two pairs of point masses and one pair of line stiffeners: (a) radiated sound power; (b) input mobility. 


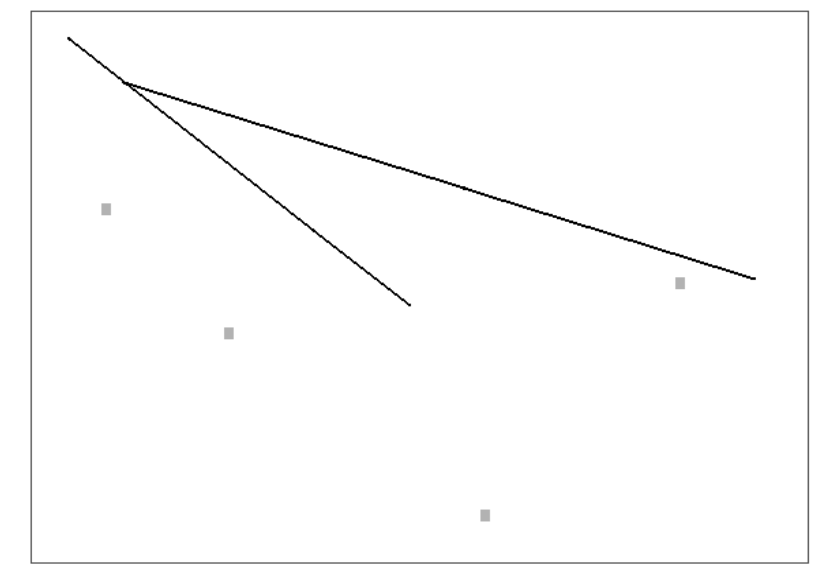

Fig. 15. Optimal location of the two stiffeners and four point masses applied to the automotive-type panel. 
(a) Radiated sound power

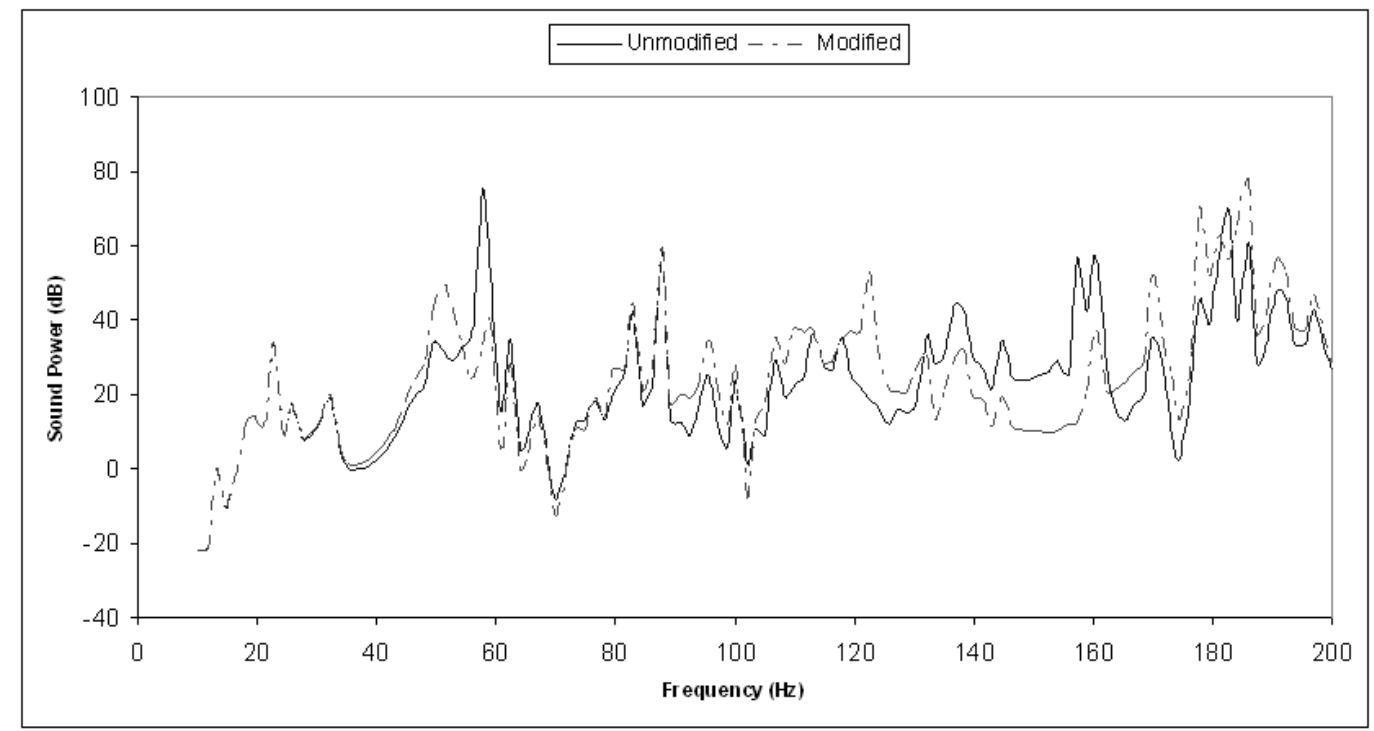

(b) Radiation efficiency

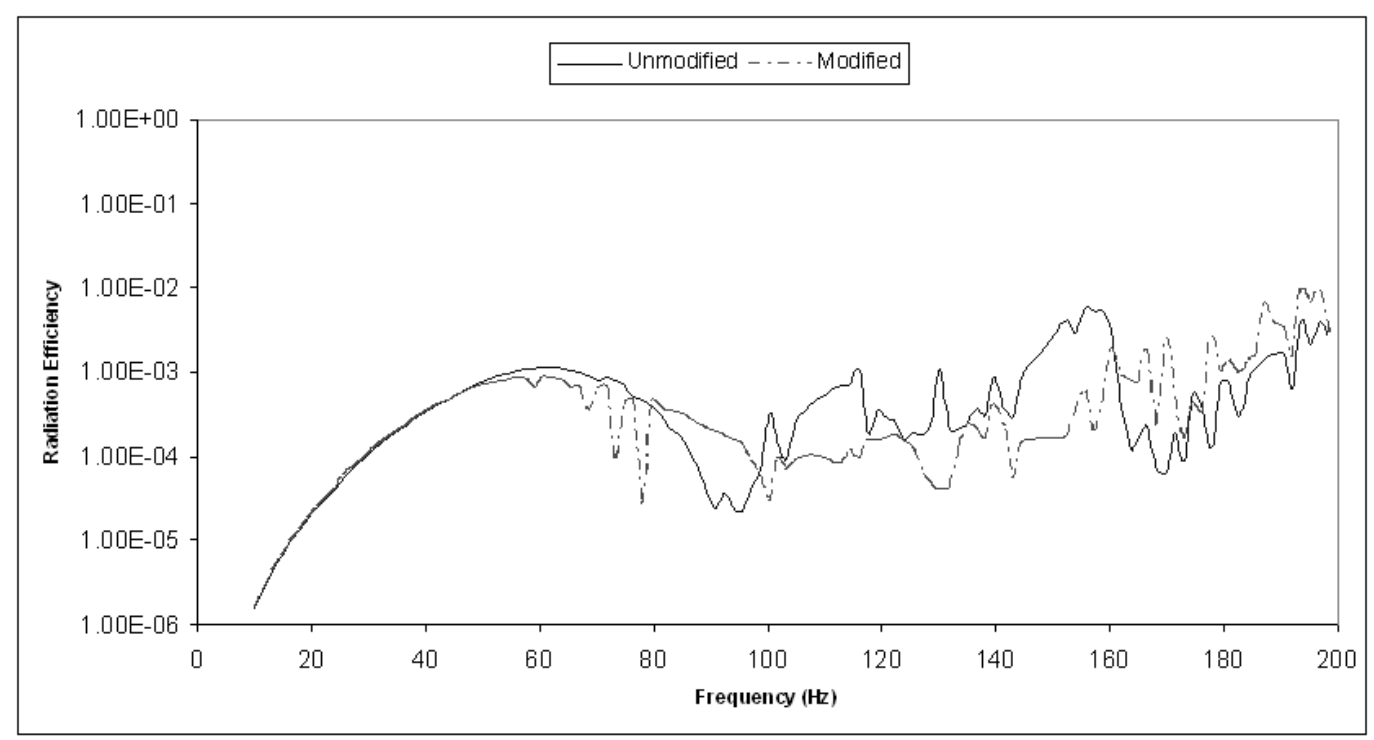

Fig. 16. Comparison of the response of the unmodified automotive-type floor panel (solid line) with the floor panel optimized with two stiffeners and four point masses (dashed line): (a) radiated sound power; (b) radiation efficiency. 